\title{
On the distribution estimation of power threshold GARCH processes
}

\author{
Esmeralda Gonçalves ${ }^{* a}$, Joana Leite ${ }^{\mathrm{b}}$ and Nazaré Mendes-Lopes ${ }^{\mathrm{a}}$

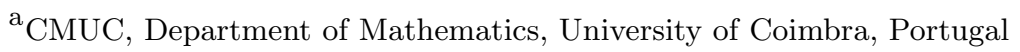 \\ ${ }^{\mathrm{b}} \mathrm{CMUC}$, Instituto Politécnico de Coimbra, ISCAC, Portugal
}

November 19, 2015

\begin{abstract}
The aim of this paper is to estimate the probability distribution of power TGARCH processes by establishing bounds for their finite dimensional laws. These bounds only depend on the parameters of the model and on the distribution function of its independent generating process. The application of this study to some particular models allows us to conjecture that this procedure is an adequate alternative to the corresponding estimation using the empirical distribution functions, particularly useful in the development of control charts for this kind of models.
\end{abstract}

Keywords: Finite dimensional laws; threshold GARCH processes.

\section{Introduction}

The knowledge of the true theoretical law of conditional heteroskedastic models remains an open question to which it seems difficult to answer. The most part of the analysis undertaken for these models is dedicated to the study of properties or probabilistic summaries of those laws. But the use of these models, for instance

\footnotetext{
*Corresponding author: Esmeralda Gonçalves, Department of Mathematics, University of Coimbra, Apartado 3008, EC Santa Cruz, 3001-501 Coimbra, Portugal. E-mail: esmerald@mat.uc.pt
} 
within the quality control theory, needs the assessment of the probability of certain regions depending on the process. In order to answer this problem, Pawlak and Schmid (2001), Gonçalves and Mendes-Lopes (2007) and Gonçalves, Leite and Mendes-Lopes (2013a) developed studies to find bounds for the finite dimensional laws of certain transformations of ARCH and TGARCH processes. These authors showed for some particular situations the usefulness of this methodology in the evaluation of control charts for conditional heteroskedastic models.

For a real stochastic process $X=\left(X_{t}, t \in \mathbb{Z}\right)$ let us define $X_{t}^{+}=\max \left(X_{t}, 0\right)$, $X_{t}^{-}=\max \left(-X_{t}, 0\right)$ and $\underline{X}_{t}$ the sigma field generated by $X_{t}, X_{t-1}, \ldots$

The process $X$ follows a power $\delta$ generalized threshold autoregressive conditionally heteroskedastic model with orders $p$ and $q$, denoted $\delta$-TGARCH $(p, q)$, if for real constants $\alpha_{0}>0, \alpha_{i} \geq 0, \beta_{i} \geq 0, \gamma_{j} \geq 0,(i=1, \ldots, p, j=1, \ldots, q)$ and a sequence of independent and identically distributed real random variables, $\left(Z_{t}, t \in \mathbb{Z}\right)$, with zero mean, unit variance and $Z_{t}$ independent of $\underline{X}_{t-1}$ we have, for every $t \in \mathbb{Z}$,

$$
\left\{\begin{array}{l}
X_{t}=\sigma_{t} Z_{t} \\
\sigma_{t}^{\delta}=\alpha_{0}+\sum_{i=1}^{p}\left[\alpha_{i}\left(X_{t-i}^{+}\right)^{\delta}+\beta_{i}\left(X_{t-i}^{-}\right)^{\delta}\right]+\sum_{j=1}^{q} \gamma_{j} \sigma_{t-j}^{\delta},
\end{array}\right.
$$

with $\delta \neq 0$, provided the following convention is considered for $\delta<0:\left(X_{t}^{+}\right)^{\delta}=0$ if $X_{t}<0$ and $\left(X_{t}^{-}\right)^{\delta}=0$ if $X_{t}>0$. The process $Z=\left(Z_{t}, t \in \mathbb{Z}\right)$ is called the generating process of $X$. If $\gamma_{j}=0, j=1, \ldots, q$, we say that $X$ follows a $\delta$ - $\operatorname{TARCH}(p)$ model.

This class of models includes the more significant and useful conditional heteroskedastic models present in literature like GARCH and GTARCH (Gonçalves, Leite and Mendes-Lopes, 2012; Pan, Wang and Tong, 2008).

We consider in the following that $\sum_{j=1}^{q} \gamma_{j}<1$ which is a necessary condition of strict and weak stationarity of $X$ and also to its stationarity up to the $\delta$-order (Gonçalves, Leite and Mendes-Lopes, 2012). Moreover, under this condition $\sigma_{t}$ is $\underline{X}_{t-1}$-measurable.

The main characteristic of threshold conditionally heteroskedastic models is the fact that they allow to take into account different reactions in the volatility according to the sign of the process values even for values with the same absolute size. So, these models capture the so-called leverage effect very common in financial 
time series of daily returns (Francq and Zakoïan, 2010). The introduction of the exponent allows to take into account long memory in the shocks of the conditional variance (Ding, Granger and Engle, 1993). A not so very common, but yet still present characteristic in some daily returns series is skewness, positive in some cases and negative in others (Taylor, 2007). We note that $X$ has a symmetric marginal distribution (relatively to the origin) if and only if the same happens to its generating process $Z$.

We point out this relation between the symmetry of the marginal distribution of a $\delta$-TGARCH process and the symmetry of its generating process to stress the importance of studying the distributions of the $\delta$-TGARCH process and not only the distributions of some transformations of these processes as Pawlak and Schmid (2001) did for the squared of a GARCH process. The transformation considered by these authors for the GARCH process only produces conclusions for the process itself if the marginal distribution of the generating process is symmetric.

In this paper we propose bounds for the finite dimension laws of the process $X$ following a $\delta$-TGARCH$(p, q)$ model with $\delta>0$ and, whenever possible, with $\delta<0$. These bounds are expressed in terms of the distribution function of the independent generating process, $Z$, and it becomes clear that the marginal law of $X$ is, in certain regions, strongly controlled by that of the process $Z$. This fact is rather relevant as we know that these laws have in general quite different characteristics; for example, the marginal law of $X$ is leptokurtic even if it doesn't happen with that of the independent generating process.

We remark that the regions where the bounds are valid seem to be larger than those theoretically stated, as suggested by the simulation studies done to evaluate the quality of those bounds.

The study here developed strongly enlarges the results of Pawlak and Schmid (2001), Gonçalves and Mendes-Lopes (2007) and Gonçalves, Leite and MendesLopes (2013a) and, so far as we know, there are no other results on this subject. In fact we consider a wide class of general conditionally heteroskedastic models and we establish bounds for the distribution of the finite dimensional laws of the process $X$.

In Section 2 we study the marginal distribution of $X$. Section 3 begins with a preliminary bound for the distribution function of the $2 n$-dimensional vector 
$\left(X_{1}^{+}, X_{1}^{-}, \ldots, X_{n}^{+}, X_{n}^{-}\right)$, which reveals useful in the bounding of the joint marginal distributions of $X$ established in the last part of this Section. In Section 4 we illustrate the overall good quality of the theoretical bounds obtained by means of a simulation study with $n=2$. In Section 5 a contribution is given for the theoretical evaluation of the run length of control charts for $\delta$-TGARCH processes with the possibility of asymmetric limits. In the last Section some concluding remarks are included.

\section{Bounds for the marginal laws}

Let $X=\left(X_{t}, t \in \mathbb{Z}\right)$ be a $\delta$-TGARCH $(p, q)$ process, $\delta \neq 0$, and let us denote by $F_{X_{t}}$ the distribution function of $X_{t}$ and by $F_{Z}$ the distribution function of $Z_{t}$.

Defining $\theta=\alpha_{0}^{\frac{1}{\delta}}\left(1-\sum_{j=1}^{q} \gamma_{j}\right)^{-\frac{1}{\delta}}$, we have $\sigma_{t}^{\delta} \geq \theta^{\delta}$, for $\delta \neq 0$. In fact, since $\gamma_{j}>0, j=1, \ldots, q$, and $\sum_{j=1}^{q} \gamma_{j}<1$ we have

$$
\left(1-\sum_{j=1}^{q} \gamma_{j} z^{j}\right)^{-1}=\sum_{j=0}^{+\infty} \varphi_{j} z^{j}
$$

with $\varphi_{j} \geq 0$ for all $j$. It follows that

$\sigma_{t}^{\delta}=\left(1-\sum_{j=1}^{q} \gamma_{j} B^{j}\right)^{-1}\left(\alpha_{0}+\sum_{i=1}^{p}\left[\alpha_{i}\left(X_{t-i}^{+}\right)^{\delta}+\beta_{i}\left(X_{t-i}^{-}\right)^{\delta}\right]\right) \geq\left(1-\sum_{j=1}^{q} \gamma_{j} B^{j}\right)^{-1} \alpha_{0}=\theta^{\delta}$.

Thus $\sigma_{t} \geq \theta$, if $\delta>0$, and $\sigma_{t} \leq \theta$, if $\delta<0$.

In consequence, and taking into account that $F_{X_{t}}(x)=E\left[F_{Z}\left(\frac{x}{\sigma_{t}}\right)\right]$, the following bounds are easily obtained:

a) for $x \geq 0$, we have

- if $\delta>0, F_{X_{t}}(x) \leq F_{Z}\left(\frac{x}{\theta}\right)$,

- if $\delta<0, F_{X_{t}}(x) \geq F_{Z}\left(\frac{x}{\theta}\right)$;

b) for $x<0$,

- if $\delta>0, F_{X_{t}}(x) \geq F_{Z}\left(\frac{x}{\theta}\right)$,

- if $\delta<0, F_{X_{t}}(x) \leq F_{Z}\left(\frac{x}{\theta}\right)$. 
To complete the bounding of $F_{X_{t}}$ we must consider some additional assumptions. Namely, we suppose that the generator process is $\delta$-integrable, that is $E\left(\left|Z_{0}\right|^{\delta}\right)<+\infty$, and

$$
S_{\delta}=\sum_{i=1}^{p}\left(\alpha_{i} \phi_{1, \delta}+\beta_{i} \phi_{2, \delta}\right)+\sum_{i=1}^{q} \gamma_{i}<1,
$$

where $E\left[\left(Z_{t}^{+}\right)^{\delta}\right]=\phi_{1, \delta}$ and $E\left[\left(Z_{t}^{-}\right)^{\delta}\right]=\phi_{2, \delta}$.

Under these assumptions, $X$ is strict and weakly stationary of order $\delta$ (Gonçalves, Leite and Mendes-Lopes, 2012); moreover, $E\left(\sigma_{t}^{\delta}\right)$ is finite, independent of $t$ and equal to

$$
E\left(\sigma_{t}^{\delta}\right)=\frac{\alpha_{0}}{1-S_{\delta}}
$$

In addition, we suppose that $\left(Z_{t}\right)$ are absolutely continuous random variables with a differentiable probability density $f_{Z}$.

The following result may then be established.

Theorem 1. Supposing $X$ a $\delta$-TGARCH $(p, q)$ under the previous assumptions and, considering the function

$$
h_{\delta}(x, y)=(1+\delta) f_{Z}\left(\frac{x}{y^{\frac{1}{\delta}}}\right)+\frac{x}{y^{\frac{1}{\delta}}} f_{Z}^{\prime}\left(\frac{x}{y^{\frac{1}{\delta}}}\right),
$$

where $y \in\left[\theta^{\delta},+\infty[\right.$, we have

a) if $x \geq 0$,

$$
\begin{aligned}
& \text { (a1) } h_{\delta}(x, y) \geq 0, \forall y \in\left[\theta^{\delta},+\infty\left[\Longrightarrow F_{X_{t}}(x) \geq F_{Z}\left(\frac{x}{\left[E\left(\sigma_{t}^{\delta}\right)\right]^{\frac{1}{\delta}}}\right)\right.\right. \\
& \text { (a2) } h_{\delta}(x, y) \leq 0, \forall y \in\left[\theta^{\delta},+\infty\left[\Longrightarrow F_{X_{t}}(x) \leq F_{Z}\left(\frac{x}{\left[E\left(\sigma_{t}^{\delta}\right)\right]^{\frac{1}{\delta}}}\right)\right.\right.
\end{aligned}
$$

b) if $x<0$,

$$
\begin{aligned}
& \text { (b1) } h_{\delta}(x, y) \geq 0, \forall y \in\left[\theta^{\delta},+\infty\left[\Longrightarrow F_{X_{t}}(x) \leq F_{Z}\left(\frac{x}{\left[E\left(\sigma_{t}^{\delta}\right)\right]^{\frac{1}{\delta}}}\right),\right.\right. \\
& \text { (b2) } h_{\delta}(x, y) \leq 0, \forall y \in\left[\theta^{\delta},+\infty\left[\Longrightarrow F_{X_{t}}(x) \geq F_{Z}\left(\frac{x}{\left[E\left(\sigma_{t}^{\delta}\right)\right]^{\frac{1}{\delta}}}\right) .\right.\right.
\end{aligned}
$$


Proof. We restrict ourselves to the proof of the conditions in a) as those referred in b) are analogously obtained.

For $t \in \mathbb{Z}$ and $x \in \mathbb{R}$, we may write $F_{X_{t}}(x)=E\left[F_{Z}\left(\frac{x}{\left(\sigma_{t}^{\delta}\right)^{\frac{1}{\delta}}}\right)\right]$. For $x \in \mathbb{R}$ arbitrarily fixed, let us consider the function $R_{\delta}:\left[\theta^{\delta},+\infty[\longrightarrow[0,1]\right.$ such that $R_{\delta}(y)=F_{Z}\left(\frac{x}{y^{\frac{1}{\delta}}}\right)$ for which we have

$$
\frac{d R_{\delta}}{d y}(y)=-\frac{x}{\delta y^{\frac{1}{\delta}+1}} f_{Z}\left(\frac{x}{y^{\frac{1}{\delta}}}\right)
$$

and

$$
\frac{d^{2} R_{\delta}}{d y^{2}}(y)=\frac{(1+\delta) x}{\delta^{2} y^{\frac{1}{\delta}+2}} f_{Z}\left(\frac{x}{y^{\frac{1}{\delta}}}\right)+\frac{x^{2}}{\delta^{2} y^{\frac{2}{\delta}+2}} f_{Z}^{\prime}\left(\frac{x}{y^{\frac{1}{\delta}}}\right)=\frac{x}{\delta^{2} y^{\frac{1}{\delta}+2}} h_{\delta}(x, y) .
$$

As referred above, under the previous hypotheses, $E\left(\sigma_{t}^{\delta}\right)$ exists and is independent of $t$. As for $x \geq 0, y \geq \theta^{\delta}$ and $h_{\delta}(x, y) \geq 0, R_{\delta}$ is a convex function, Jensen's inequality allows to write

$$
F_{X_{t}}(x)=E\left[F_{Z}\left(\frac{x}{\left(\sigma_{t}^{\delta}\right)^{\frac{1}{\delta}}}\right)\right] \geq F_{Z}\left(\frac{x}{\left[E\left(\sigma_{t}^{\delta}\right)\right]^{\frac{1}{\delta}}}\right) .
$$

Similarly, as $R_{\delta}$ is a concave function when $x>0, y \geq \theta^{\delta}$ and $h_{\delta}(x, y) \leq 0$, we have

$$
F_{X_{t}}(x)=E\left[F_{Z}\left(\frac{x}{\left(\sigma_{t}^{\delta}\right)^{\frac{1}{\delta}}}\right)\right] \leq F_{Z}\left(\frac{x}{\left[E\left(\sigma_{t}^{\delta}\right)\right]^{\frac{1}{\delta}}}\right)
$$

From the previous results we conclude that to bound $F_{X_{t}}$ we have to discuss the sign of the function

$$
h_{\delta}(x, y)=(1+\delta) f_{Z}\left(x y^{-\frac{1}{\delta}}\right)+x y^{-\frac{1}{\delta}} f_{Z}^{\prime}\left(x y^{-\frac{1}{\delta}}\right), \forall y \in\left[\theta^{\delta},+\infty[;\right.
$$

namely, to properly combine the bounds determined in the beginning of this section and those in theorem 1 , we are interested in $h_{\delta} \leq 0$, when $\delta<0$, and $h_{\delta} \geq 0$, when $\delta>0$. In the following we illustrate this discussion for some distributions of the generator process $Z$ and some values of $\delta$. 
Example 1. Let us consider $Z_{t}$ following a standard normal distribution. For $y \in\left[\theta^{\delta},+\infty[\right.$, we have

$$
h_{\delta}(x, y)=f_{Z}\left(\frac{x}{y^{\frac{1}{\delta}}}\right)\left[(1+\delta)-\left(\frac{x}{y^{\frac{1}{\delta}}}\right)^{2}\right], x \in \mathbb{R} .
$$

So, it is obvious that $\forall x \in \mathbb{R}, h_{\delta}(x, y) \leq 0$ if $\delta \leq-1$.

Let us consider now $-1<\delta<0$. As $h_{\delta}(x, y) \leq 0 \Leftrightarrow|x| \geq y^{\frac{1}{\delta}} \sqrt{1+\delta}$ and $y^{\frac{1}{\delta}} \leq \theta$, we may assure that $h_{\delta}(x, y) \leq 0$ at least for $\left.\left.x \in\right]-\infty,-\theta \sqrt{1+\delta}\right] \cup$ $[\theta \sqrt{1+\delta},+\infty[$.

Analogously we conclude that, if $\delta>0$, then $h_{\delta}(x, y) \geq 0$ at least for $x \in$ $[-\theta \sqrt{1+\delta}, \theta \sqrt{1+\delta}]$, taking into account that, in this case, $y^{\frac{1}{\delta}} \geq \theta$.

Taking into consideration these results, we present in Figure 1 the bounds of the distribution function of a $\delta$-TGARCH $(1,1)$, with $\alpha_{0}=10, \alpha_{1}=0.3, \beta_{1}=0.5$, $\gamma_{1}=0.2$ and a standard Gaussian generator process, for $\delta$ equal to $-\frac{1}{2},-\frac{1}{3}, \frac{1}{2}, 1$, $\frac{3}{2}$ and 2 , representing, in each case, the bounds $F_{Z}\left(\frac{x}{\theta}\right)$ and $F_{Z}\left(x\left[E\left(\sigma_{t}^{\delta}\right)\right]^{-\frac{1}{\delta}}\right)$ and the empirical estimation of $F_{X_{t}}(x)$. This empirical distribution was obtained by a simulation study considering a sample of 10000 observations of the $\delta$-TGARCH $(1,1)$ process $X$.

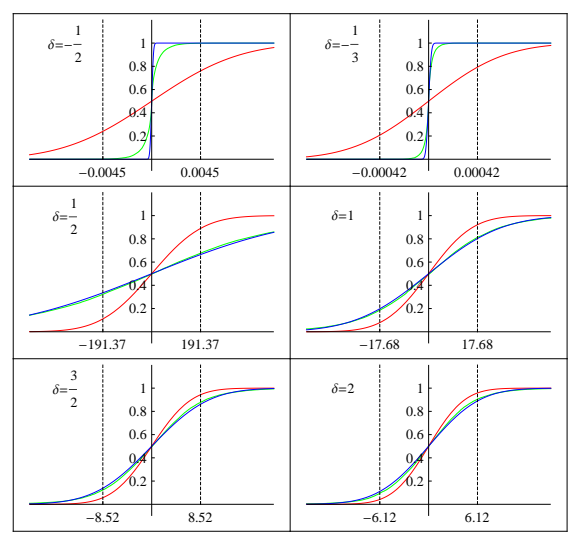

Figure 1: Plots of $F_{Z}\left(\frac{x}{\theta}\right)$ (red), $F_{Z}\left(x\left[E\left(\sigma_{t}^{\delta}\right)\right]^{-\frac{1}{\delta}}\right)$ (blue) and the estimate of $F_{X_{t}}(x)$ (green), for $X \sim \delta$-TGARCH $(1,1)$ and $Z_{t} \sim N(0,1)$

According with the previous study these bounds are theoretically validated for $x$ between the lines $x= \pm \theta \sqrt{1+\delta}$, when $\delta>0$, and outside of these lines, 
when $\delta<0$. Nevertheless, the previous plots lead us to conjecture that these bounds are still valid for $x$ outside those intervals, which is understandable since our results only establish sufficient conditions for the validity of these bounding; moreover, the quality of the bounds seems to be strongly related to the corresponding value of $S_{\delta}$ (for the chosen increasing values of $\delta, S_{\delta}$ is approximately equal to $0.888,0.739,0.529,0.520,0.544$ and 0.6 , respectively). In what concerns the bounding accuracy, we stress the high quality of the bounds related to the function $F_{Z}\left(x\left[E\left(\sigma_{t}^{\delta}\right)\right]^{-\frac{1}{\delta}}\right)$ that depends on all the model parameters.

Example 2. Let us consider random variables $Z_{t}$ following a centered and reduced distribution based on the Student law with parameter $n, n>2$, that is, with density $f_{Z}(x)=\frac{1}{\sqrt{(n-2) \pi}} \frac{\Gamma\left(\frac{n+1}{2}\right)}{\Gamma\left(\frac{n}{2}\right)}\left(1+\frac{x^{2}}{n-2}\right)^{-\frac{n+1}{2}}, x \in \mathbb{R}$. So, $f_{Z}^{\prime}(x)=-\frac{x(n+1)}{n-2+x^{2}} f_{Z}(x)$, $x \in \mathbb{R}$.

As, for $y \in\left[\theta^{\delta},+\infty\left[, h_{\delta}(x, y)=f_{Z}\left(\frac{x}{y^{\frac{1}{\delta}}}\right) \frac{(1+\delta)(n-2) y^{\frac{2}{\delta}}+(\delta-n) x^{2}}{y^{\frac{2}{\delta}}(n-2)+x^{2}}, x \in \mathbb{R}\right.\right.$, we have the following discussion:

i) $h_{\delta}(x, y) \leq 0, \forall x \in \mathbb{R}$, if $\delta \leq-1$;

ii) $\left.\left.h_{\delta}(x, y) \leq 0, \forall x \in\right]-\infty,-\theta \sqrt{\frac{(1+\delta)(n-2)}{n-\delta}}\right] \cup\left[\theta \sqrt{\frac{(1+\delta)(n-2)}{n-\delta}},+\infty[\right.$, if $-1<$ $\delta<0$

iii) $h_{\delta}(x, y) \geq 0, \forall x \in\left[-\theta \sqrt{\frac{(1+\delta)(n-2)}{n-\delta}}, \theta \sqrt{\frac{(1+\delta)(n-2)}{n-\delta}}\right]$, if $0<\delta<n$;

iv) $h_{\delta}(x, y) \geq 0, \forall x \in \mathbb{R}$, if $\delta \geq n$.

As in the Example 1, a simulation study was developed considering four $\delta$ TGARCH models with generator process following the previous distribution with $n=6$, parameters $\delta=1, \alpha_{0}=10, \alpha_{1}=0.3, \beta_{1}=0.5$ and $\gamma_{1}=0.2$ and orders $(1,1),(2,1),(1,2)$ and $(2,2)$. We note that the values of $S_{\delta}$ are, respectively, 0.5 , $0.688,0.75$ and 0.938 . The bounds obtained in each one of these cases are plotted in Figure 2.

Despite being in a very different situation, the behavior of these bounds are similar to those of the previous example; in particular, we highlight that the bounding accuracy seems also to be dependent on the $S_{\delta}$ value. 


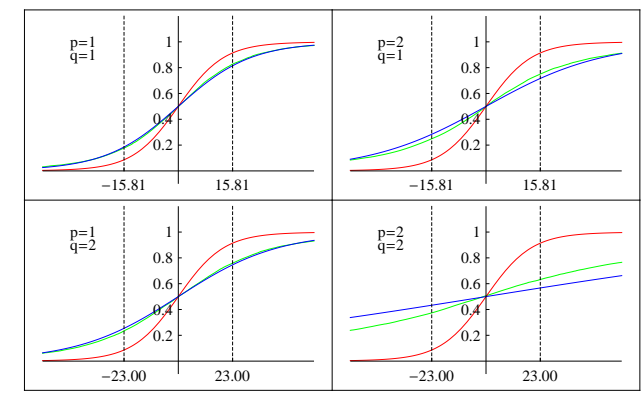

Figure 2: Plots of $F_{Z}\left(\frac{x}{\theta}\right)$ (red), $F_{Z}\left(x\left[E\left(\sigma_{t}^{\delta}\right)\right]^{-\frac{1}{\delta}}\right)$ (blue) and the estimate of $F_{X_{t}}(x)$ (green), for $X \sim 1$-TGARCH$(p, q)$ and $Z_{t}=\sqrt{\frac{2}{3}} W$, with $W \sim t_{6}$

Example 3. Let us consider a mixture of two Gaussian distributions (not necessarily symmetric), that is

$$
f_{Z}(x)=p_{1} f_{1}\left(x ; \mathfrak{m}_{1}, \mathfrak{s}_{1}\right)+p_{2} f_{2}\left(x ; \mathfrak{m}_{2}, \mathfrak{s}_{2}\right),
$$

where $\left.p_{1}, p_{2} \in\right] 0,1\left[\right.$ with $p_{1}+p_{2}=1$, and where $f_{k}\left(\cdot ; \mathfrak{m}_{k}, \mathfrak{s}_{k}\right)$ is the Gaussian probability density with mean $\mathfrak{m}_{k}$ and variance $\mathfrak{s}_{k}^{2}$.

In order to obtain a centered and reduced generator process, we have the following relations $p_{2}=1-p_{1}, \mathfrak{m}_{2}=\frac{p_{1} \mathfrak{m}_{1}}{p_{1}-1}$ and $\mathfrak{s}_{2}=\sqrt{\frac{1-p_{1}\left(\mathfrak{m}_{1}^{2}+\mathfrak{s}_{1}^{2}\right)-\left(1-p_{1}\right)\left(\frac{p_{1} \mathfrak{m}_{1}}{p_{1}-1}\right)^{2}}{1-p_{1}}}$.

To analyze the sign of the function $h_{\delta}$ we restrict ourselves to the case $\delta=1$.

As $f_{k}^{\prime}\left(x ; \mathfrak{m}_{k}, \mathfrak{s}_{k}\right)=f_{k}\left(x ; \mathfrak{m}_{k}, \mathfrak{s}_{k}\right)\left(-\frac{\left(x-\mathfrak{m}_{k}\right)}{\mathfrak{s}_{k}^{2}}\right)$, we have, for $x \in \mathbb{R}$,

$$
h_{1}(x, y)=2 f_{Z}\left(\frac{x}{y}\right)+\frac{x}{y} f_{Z}^{\prime}\left(\frac{x}{y}\right)=\sum_{k=1}^{2} p_{k} f_{k}\left(\frac{x}{y} ; \mathfrak{m}_{k}, \mathfrak{s}_{k}\right)\left[2-\frac{\left(\frac{x}{y}-\mathfrak{m}_{k}\right) \frac{x}{y}}{\mathfrak{s}_{k}^{2}}\right]
$$

and so $h_{1}(x, y) \geq 0$ if, at least, $\frac{x}{y} \in \bigcap_{k=1}^{2}\left[\frac{\mathfrak{m}_{k}-\sqrt{\mathfrak{m}_{k}^{2}+8 \mathfrak{s}_{k}^{2}}}{2}, \frac{\mathfrak{m}_{k}+\sqrt{\mathfrak{m}_{k}^{2}+8 \mathfrak{s}_{k}^{2}}}{2}\right]$, that is $\frac{x}{y} \in$ $\left[l_{1}, l_{2}\right]$, with $l_{1}=\max _{k \in\{1,2\}} \frac{\mathfrak{m}_{k}-\sqrt{\mathfrak{m}_{k}^{2}+8 \mathfrak{s}_{k}^{2}}}{2}$ and $l_{2}=\min _{k \in\{1,2\}} \frac{\mathfrak{m}_{k}+\sqrt{\mathfrak{m}_{k}^{2}+8 \mathfrak{s}_{k}^{2}}}{2}$.

As this theoretical study leads us only to a sufficient condition for the positivity of $h_{1}$, we may improve this study using numerical and graphical methods.

So, let $\left(p_{1}, \mathfrak{m}_{1, \mathfrak{s}_{1}}\right)=(0.65,-0.15,0.4)$ and $\left(p_{1}, \mathfrak{m}_{1, \mathfrak{s}_{1}}\right)=(0.6,0.3,0.4)$ be two different set of parameters, denoted $A$ and $B$ respectively, associated to the distribution considered above. In Figure 3 we plot the function $h_{1}$, for $y=1$ without loss of generality, corresponding to each one of these cases. 


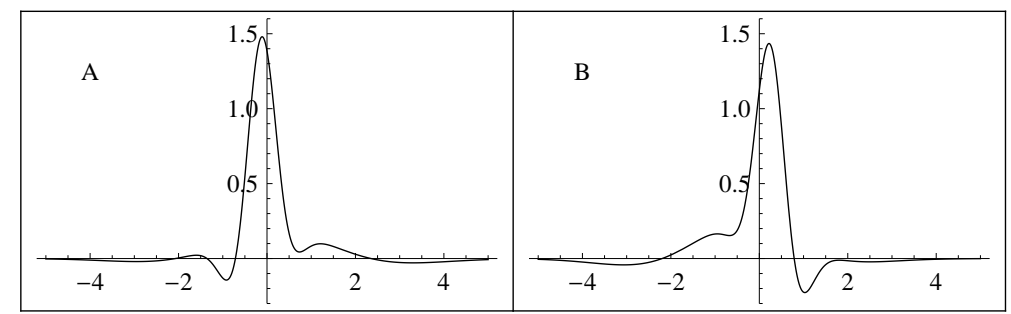

Figure 3: Function $h_{1}$ when $Z_{t}$ follows a mixture of Gaussian distributions with parameters $\mathrm{A},\left(p_{1}, \mathfrak{m}_{1, \mathfrak{s}_{1}}\right)=(0.65,-0.15,0.4)$ and $\mathrm{B},\left(p_{1}, \mathfrak{m}_{1, \mathfrak{s}_{1}}\right)=(0.6,0.3,0.4)$

Analyzing these plots and using numerical methods we conclude, in case $A$, that $h_{1}(x, y) \geq 0, \forall y \in\left[\theta,+\infty\left[\right.\right.$, if $\frac{x}{y} \in[-0.714,2.353]$ and if $x \in[-0.714 \theta, 2.353 \theta]$. Similarly, in case $B$, we have the same result if $\frac{x}{y} \in[-2.198,0.788]$ and also if $x \in$ $[-2.198 \theta, 0.788 \theta]$.

A simulation study is developed for this kind of generator process distribution, considering the laws associated to the cases of parameter sets $A$ and $B$. Unlike the previous examples we take now skewed distributions for $Z_{t}$. Namely, in cases denoted $\mathrm{A} 1$ e $\mathrm{A} 2$, we choose $\left(p_{1}, \mathfrak{m}_{1, \mathfrak{s}_{1}}\right)=(0.65,-0.15,0.4)$ (positive skewness); in cases denoted $\mathrm{B} 1 \mathrm{e} \mathrm{B} 2$, we take $\left(p_{1}, \mathfrak{m}_{1, \mathfrak{s}_{1}}\right)=(0.6,0.3,0.4)$ (negative skewness). Regarding the $\delta$-TGARCH process we consider in all the cases $\delta=1, \alpha_{0}=10$, $\alpha_{1}=0.15, \beta_{1}=0.5$ and, in A1 and B1, $\gamma_{1}=0.2$; in A2 and B2 we take $\gamma_{1}=0.6$. So, the values for $S_{\delta}$ are equal to $0.416,0.816,0.430$ and 0.830 in the cases A1, A2, B1 and B2, respectively. The corresponding plots are presented in Figure 4.

Also in this asymmetrical case, the conclusions are similar to the previous ones. Moreover, this example reinforces the conjecture that the bounding accuracy is better for smaller values of $S_{\delta}$.

Observation 1. Before ending this section, we point out that the technique used to prove theorem 1 can be extended to the log-GARCH and EGARCH models, even though the one used to obtain the initial bounds presented in this section cannot, as in these two cases there is no real positive number $\theta$ such that $\sigma_{t} \geq \theta$.

In fact, (Francq, Wintenberger and Zakoïan, 2013) a process $X$ follows a log- 


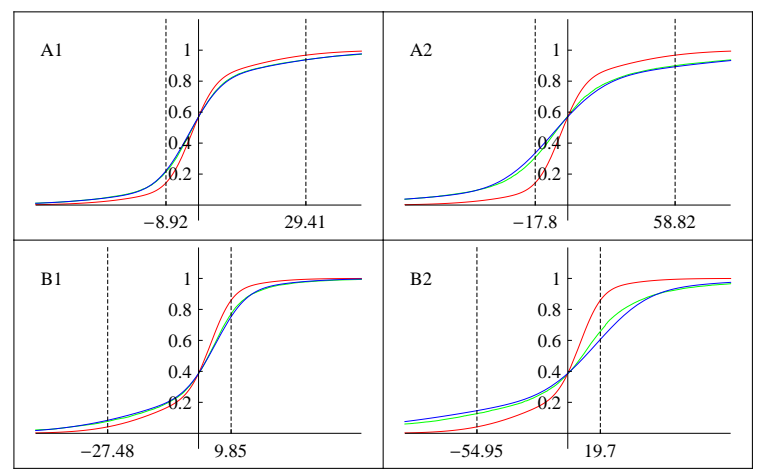

Figure 4: Plots of $F_{Z}\left(\frac{x}{\theta}\right)$ (red), $F_{Z}\left(x\left[E\left(\sigma_{t}^{\delta}\right)\right]^{-\frac{1}{\delta}}\right)$ (blue) and the estimate of $F_{X_{t}}(x)$ (green), for $X \sim 1$-TGARCH(1,1) and $Z_{t} \sim N M$

$\operatorname{GARCH}(p, q)$ model if

$$
\left\{\begin{array}{l}
X_{t}=\sigma_{t} Z_{t} \\
\log \sigma_{t}^{2}=\omega+\sum_{i=1}^{q}\left(\alpha_{i+1} \mathbb{1}_{\left\{X_{t-i}>0\right\}}+\alpha_{i-} \mathbb{1}_{\left\{X_{t-i}<0\right\}}\right) \log X_{t-i}^{2}+\sum_{j=1}^{p} \beta_{j} \log \sigma_{t-j}^{2}
\end{array}\right.
$$

and follows a $\operatorname{EGARCH}(p, l)$ model if

$$
\left\{\begin{array}{l}
X_{t}=\sigma_{t} Z_{t} \\
\log \sigma_{t}^{2}=\omega+\sum_{j=1}^{p} \beta_{j} \log \sigma_{t-j}^{2}+\sum_{k=1}^{l}\left(\gamma_{k} Z_{t-k}+\delta_{k}\left|Z_{t-k}\right|\right)
\end{array}\right.
$$

where $\sigma_{t}>0$ but without positivity constrains imposed to the parameters.

Regarding the procedure adaptation related to theorem 1 , it is easily done if we note that, because $\log \left|X_{t}\right|=\log \sigma_{t}+\log \left|Z_{t}\right|$, we have

$$
F_{\log \left|X_{t}\right|}(x)=E\left[F_{\left|Z_{t}\right|}\left(\exp \left(x-\log \sigma_{t}\right)\right)\right] .
$$

Assuming, for simplicity, that $Z_{t}$ are symmetrically distributed, then

$$
F_{\log \left|X_{t}\right|}(x)=2 E\left[F_{Z}\left(\exp \left(x-\log \sigma_{t}\right)\right)\right]-1
$$

and, because $X_{t}$ are also symmetrically distributed $F_{\log \left|X_{t}\right|}(x)=2 F_{X_{t}}(\exp x)-1$, we have

$$
F_{X_{t}}(\exp x)=E\left[F_{Z}\left(\exp \left(x-\log \sigma_{t}\right)\right)\right] .
$$

So, for $x$ arbitrarily fixed, the function $R$ in the proof of theorem 1 , is defined, in the case of these two models, by $R:]-\infty,+\infty[\longrightarrow[0,1]$ such that 
$R(y)=F_{Z}(\exp (x-y))$. From where it is easily deduced that

$$
h(x, y)=f_{Z}(\exp (x-y))+\exp (x-y) f_{Z}^{\prime}(\exp (x-y)) .
$$

Consequently, if $h(x, y) \geq 0$, for all $y \in \mathbb{R}$, then

$$
F_{X_{t}}(\exp x) \geq F_{Z}\left(\exp \left(x-E\left(\log \sigma_{t}\right)\right)\right)-1
$$

and if $h(x, y) \leq 0$, for all $y \in \mathbb{R}$, then

$$
F_{X_{t}}(\exp x) \leq F_{Z}\left(\exp \left(x-E\left(\log \sigma_{t}\right)\right)\right)-1 .
$$

For example, in Francq, Wintenberger and Zakoïan (2013), the value of $E\left(\log \sigma_{t}\right)$ is available in the case of the stationary model $\log$ - $\mathrm{GARCH}(1,1)$ with symmetrically distributed generating process.

\section{Bounds for the finite dimensional laws}

In this section we concentrate our study on $\delta$-TGARCH processes with positive power $\delta$ and generator process with absolutely continuous distribution. To develop bounds for the distribution function of the finite dimensional laws of the process, we begin by an auxiliary study on the bounding of the distribution function of the vector $\left(X_{1}^{+}, X_{1}^{-}, \ldots, X_{n}^{+}, X_{n}^{-}\right)$.

\subsection{Bounds for the distribution of $\left(X_{1}^{+}, X_{1}^{-}, \ldots, X_{n}^{+}, X_{n}^{-}\right)$}

Let $X=\left(X_{t}, t \in \mathbb{Z}\right)$ be a $\delta$-TGARCH$(p, q)$ process, $\delta>0$, for which the law of $Z_{t}$ is absolutely continuous.

As in Gonçalves, Leite and Mendes-Lopes (2013a), it is easy to establish the following upper bound for the distribution function of $\left(X_{1}^{+}, X_{1}^{-}, \ldots, X_{n}^{+}, X_{n}^{-}\right)$:

$$
\begin{aligned}
& \forall\left(x_{1}, x_{1}^{*}, \ldots, x_{n}, x_{n}^{*}\right) \in\left(\mathbb{R}_{0}^{+}\right)^{2 n}, n \in \mathbb{N}, \\
& \quad F_{\left(X_{1}^{+}, X_{1}^{-}, \ldots, X_{n}^{+}, X_{n}^{-}\right)}\left(x_{1}, x_{1}^{*}, \ldots, x_{n}, x_{n}^{*}\right) \leq \prod_{t=1}^{n}\left[F_{Z}\left(\frac{x_{t}}{\theta}\right)-F_{Z}\left(-\frac{x_{t}^{*}}{\theta}\right)\right] .
\end{aligned}
$$

In fact, taking into account the definitions of $X_{t}^{+}$and $X_{t}^{-}$and the positivity of $\sigma_{t}$, we have

$$
F_{\left(X_{1}^{+}, X_{1}^{-}, \ldots, X_{n}^{+}, X_{n}^{-}\right)}\left(x_{1}, x_{1}^{*}, \ldots, x_{n}, x_{n}^{*}\right)=P\left(-\frac{x_{t}^{*}}{\sigma_{t}} \leq Z_{t} \leq \frac{x_{t}}{\sigma_{t}}, t=1, \ldots, n\right) .
$$


So, as $\sigma_{t}>\theta$ and $Z_{t}$ are i.i.d. and absolutely continuous random variables, the announced result follows from the inequality

$$
\begin{aligned}
P\left(-\frac{x_{t}^{*}}{\sigma_{t}} \leq Z_{t} \leq \frac{x_{t}}{\sigma_{t}}, t=1, \ldots, n\right) & \leq P\left(-\frac{x_{t}^{*}}{\theta} \leq Z_{t} \leq \frac{x_{t}}{\theta}, t=1, \ldots, n\right) \\
& =\prod_{t=1}^{n}\left[F_{Z}\left(\frac{x_{t}}{\theta}\right)-F_{Z}\left(-\frac{x_{t}^{*}}{\theta}\right)\right] .
\end{aligned}
$$

We note that, when $\delta$ is negative, a reversed inequality is obtained using the same technique.

As previously, the methodology used to obtain the other bound is more refined and leads us to a precise approximation of the distribution function under study. However, it is only valid for positive $\delta$.

Let us begin by considering the following result.

Lemma 1. Under the previous conditions, we have

$$
P\left(V_{t} \leq Z_{t} \leq W_{t}, t=1, \ldots, k\right)=\prod_{t=1}^{k} E\left[F_{Z}\left(W_{t}\right)-F_{Z}\left(V_{t}\right)\right],
$$

where $V_{t}$ and $W_{t}$, such that $V_{t} \leq W_{t}$, are $\underline{X}_{0}$-measurable random variables, $t$ $\in\{1, \ldots, k\}$.

Proof. Using expectation and conditional expectation properties and taking into account that $Z_{1}, \ldots, Z_{k}$ are i.i.d. absolutely continuous random variables and also that they are independent from $\underline{X}_{0}$, it is easy to conclude that

$$
\begin{aligned}
P\left(V_{t} \leq Z_{t} \leq W_{t}, t=1, \ldots, k\right) & =E\left[E\left(\mathbb{1}_{\left[V_{1}, W_{1}\right] \times \ldots \times\left[V_{k}, W_{k}\right]}\left(Z_{1}, \ldots, Z_{k}\right) \mid \underline{X}_{0}\right)\right] \\
& =\prod_{t=1}^{k} E\left[F_{Z}\left(W_{t}\right)-F_{Z}\left(V_{t}\right)\right] .
\end{aligned}
$$

In the next theorem we present the required lower bound. For simplicity, we restrict the presentation of this study to $\delta$ - $\operatorname{TARCH}(p)$ (that is, $\gamma_{1}=\ldots=\gamma_{q}=0$ ) and $\delta$-TGARCH $(1,1)$ models; more general cases use the same procedure with a more complicated framework.

Theorem 2. Let $X$ be a $\delta$-TGARCH$(p, q)$ process, $\delta>0$, such that $E\left(\left|Z_{0}\right|^{\delta}\right)<$ $+\infty$ and $S_{\delta}<1$. Supposing $Z_{t}$ absolutely continuous with a differentiable density $f_{Z}$, let us define, for each $y \in\left[\theta^{\delta},+\infty[\right.$, the function 


$$
h_{\delta}(x, y)=(1+\delta) f_{Z}\left(\frac{x}{y^{\frac{1}{\delta}}}\right)+\frac{x}{y^{\frac{1}{\delta}}} f_{Z}^{\prime}\left(\frac{x}{y^{\frac{1}{\delta}}}\right)
$$

For $\left(x_{1}, x_{1}^{*}, \ldots, x_{n}, x_{n}^{*}\right) \in\left(\mathbb{R}_{0}^{+}\right)^{2 n}$ such that

$$
x_{t} h_{\delta}\left(x_{t}, y\right)+x_{t}^{*} h_{\delta}\left(-x_{t}^{*}, y\right) \geq 0, \forall y \in\left[\theta^{\delta},+\infty[,\right.
$$

where $t=\left\{\begin{array}{ll}1, \ldots, \min \{p, n\} & \text { if } q=0 \\ 1, \ldots, n, & \text { if } q=1\end{array}\right.$, we have:

a) if $q=0$ and

$$
\begin{aligned}
& \text { (a1) } 1 \leq p \leq n \text {, } \\
& F_{\left(X_{1}^{+}, X_{1}^{-}, \ldots, X_{n}^{+}, X_{n}^{-}\right)}\left(x_{1}, x_{1}^{*}, \ldots, x_{n}, x_{n}^{*}\right) \geq \\
& \geq \prod_{t=1}^{p}\left[F_{Z}\left(\frac{x_{t}}{u_{t}^{1 / \delta}}\right)-F_{Z}\left(-\frac{x_{t}^{*}}{u_{t}^{1 / \delta}}\right)\right] \prod_{t=p+1}^{n}\left[F_{Z}\left(\frac{x_{t}}{v_{t}^{1 / \delta}}\right)-F_{Z}\left(-\frac{x_{t}^{*}}{v_{t}^{1 / \delta}}\right)\right],
\end{aligned}
$$

(a2) $p>n \geq 1$

$$
\begin{aligned}
& F_{\left(X_{1}^{+}, X_{1}^{-}, \ldots, X_{n}^{+}, X_{n}^{-}\right)}\left(x_{1}, x_{1}^{*}, \ldots, x_{n}, x_{n}^{*}\right) \geq \prod_{t=1}^{n}\left[F_{Z}\left(\frac{x_{t}}{u_{t}^{1 / \delta}}\right)-F_{Z}\left(-\frac{x_{t}^{*}}{u_{t}^{1 / \delta}}\right)\right], \\
& \text { where } u_{t}=\sum_{i=1}^{t-1}\left[\alpha_{i}\left(x_{t-i}\right)^{\delta}+\beta_{i}\left(x_{t-i}^{*}\right)^{\delta}\right]+E\left(\sigma_{t}^{\delta}\right)\left[1-\sum_{i=1}^{t-1}\left(\alpha_{i} \phi_{1, \delta}+\beta_{i} \phi_{2, \delta}\right)\right] \\
& \text { and } v_{t}=\alpha_{0}+\sum_{i=1}^{p}\left[\alpha_{i}\left(x_{t-i}\right)^{\delta}+\beta_{i}\left(x_{t-i}^{*}\right)^{\delta}\right]
\end{aligned}
$$

b) if $p=1$ and $q=1$,

$$
\begin{gathered}
F_{\left(X_{1}^{+}, X_{1}^{-}, \ldots, X_{n}^{+}, X_{n}^{-}\right)}\left(x_{1}, x_{1}^{*}, \ldots, x_{n}, x_{n}^{*}\right) \geq \prod_{t=1}^{n}\left[F_{Z}\left(\frac{x_{t}}{w_{t}^{1 / \delta}}\right)-F_{Z}\left(-\frac{x_{t}^{*}}{w_{t}^{1 / \delta}}\right)\right], \\
\text { where } w_{t}=\alpha_{0} \sum_{j=1}^{t-1} \gamma_{1}^{j-1}+\sum_{j=1}^{t-1} \gamma_{1}^{j-1}\left[\alpha_{1}\left(x_{t-j}\right)^{\delta}+\beta_{1}\left(x_{t-j}^{*}\right)^{\delta}\right]+\gamma_{1}^{t-1} E\left(\sigma_{t}^{\delta}\right) .
\end{gathered}
$$

Proof. Let $\left(x_{1}, x_{1}^{*}, \ldots, x_{n}, x_{n}^{*}\right) \in\left(\mathbb{R}_{0}^{+}\right)^{2 n}$. We introduce the random variables $\widetilde{U}_{t}=$ $\alpha_{0}+\sum_{i=1}^{t-1}\left[\alpha_{i}\left(x_{t-i}\right)^{\delta}+\beta_{i}\left(x_{t-i}^{*}\right)^{\delta}\right]+\sum_{i=t}^{p}\left[\alpha_{i}\left(X_{t-i}^{+}\right)^{\delta}+\beta_{i}\left(X_{t-i}^{-}\right)^{\delta}\right], t \in\{1, \ldots, n\}$.

(a1) Let us begin by considering $q=0$ and $1 \leq p \leq n$.

As $\delta>0$, if $X_{t}^{+} \leq x_{t}$ and $X_{t}^{-} \leq x_{t}^{*}, t \in\{1, \ldots, n\}$, we have 
- if $t \in\{2, \ldots, p\}$,

$$
\begin{aligned}
\sigma_{t}^{\delta} & =\alpha_{0}+\sum_{i=1}^{t-1}\left[\alpha_{i}\left(X_{t-i}^{+}\right)^{\delta}+\beta_{i}\left(X_{t-i}^{-}\right)^{\delta}\right]+\sum_{i=t}^{p}\left[\alpha_{i}\left(X_{t-i}^{+}\right)^{\delta}+\beta_{i}\left(X_{t-i}^{-}\right)^{\delta}\right] \\
& \leq \widetilde{U}_{t}
\end{aligned}
$$

as for $i \in\{1, \ldots, t-1\}, 1 \leq t-i \leq p-1<n$ and for $i \in\{t, \ldots, p\}, t-i \leq 0$;

- if $t=1, \sigma_{1}^{\delta}=\alpha_{0}+\sum_{i=1}^{p}\left[\alpha_{i}\left(X_{1-i}^{+}\right)^{\delta}+\beta_{i}\left(X_{1-i}^{-}\right)^{\delta}\right]$, thus $\sigma_{1}=\widetilde{U}_{1}$;

- and, if $t \in\{p+1, \ldots, n\}$,

$$
\begin{aligned}
\sigma_{t}^{\delta} & =\alpha_{0}+\sum_{i=1}^{p}\left[\alpha_{i}\left(X_{t-i}^{+}\right)^{\delta}+\beta_{i}\left(X_{t-i}^{-}\right)^{\delta}\right] \\
& \leq \alpha_{0}+\sum_{i=1}^{p}\left[\alpha_{i}\left(x_{t-i}\right)^{\delta}+\beta_{i}\left(x_{t-i}^{*}\right)^{\delta}\right]=v_{t},
\end{aligned}
$$

as, for $i \in\{1, \ldots, p\}, 1 \leq t-i \leq n-1$.

Using the definitions of $X_{t}^{+}$and $X_{t}^{-}$, the previous bounds and lemma, and taking into consideration that $\widetilde{U}_{t}^{\frac{1}{\delta}}, t=1, \ldots, p$, and $v_{t}^{\frac{1}{\delta}}, t=p+1, \ldots, n$, are $\underline{X}_{0^{-}}$ measurable, we are able to write

$$
\begin{aligned}
& F_{\left(X_{1}^{+}, X_{1}^{-}, \ldots, X_{n}^{+}, X_{n}^{-}\right)}\left(x_{1}, x_{1}^{*}, \ldots, x_{n}, x_{n}^{*}\right) \geq \\
& \quad \geq P\left(-\frac{x_{t}^{*}}{\widetilde{U}_{t}^{\frac{1}{\delta}}} \leq Z_{t} \leq \frac{x_{t}}{\widetilde{U}_{t}^{\frac{1}{\delta}}}, t=1, \ldots, p,-\frac{x_{t}^{*}}{v_{t}^{\frac{1}{\delta}}} \leq Z_{t} \leq \frac{x_{t}}{v_{t}^{\frac{1}{\delta}}}, t=p+1, \ldots, n\right) \\
& \quad=\prod_{t=1}^{p} E\left[F_{Z}\left(\frac{x_{t}}{\widetilde{U}_{t}^{\frac{1}{\delta}}}\right)-F_{Z}\left(-\frac{x_{t}^{*}}{\widetilde{U}_{t}^{\frac{1}{\delta}}}\right)\right] \prod_{t=p+1}^{n}\left[F_{Z}\left(\frac{x_{t}}{v_{t}^{\frac{1}{\delta}}}\right)-F_{Z}\left(-\frac{x_{t}^{*}}{v_{t}^{\frac{1}{\delta}}}\right)\right] .
\end{aligned}
$$

For $a_{1}$ and $a_{2}$ arbitrarily fixed in $\mathbb{R}_{0}^{+}$and $\mathbb{R}_{0}^{-}$, respectively, let us consider the function $\mathcal{R}_{\delta}:\left[\theta^{\delta},+\infty[\longrightarrow[-1,1]\right.$ defined by

$$
\mathcal{R}_{\delta}(y)=F_{Z}\left(\frac{a_{1}}{y^{\frac{1}{\delta}}}\right)-F_{Z}\left(\frac{a_{2}}{y^{\frac{1}{\delta}}}\right) .
$$

It is easy to show that

$$
\frac{d^{2} \mathcal{R}_{\delta}}{d y^{2}}(y)=\frac{1}{\delta^{2} y^{\frac{1}{\delta}+2}}\left[a_{1} h_{\delta}\left(a_{1}, y\right)-a_{2} h_{\delta}\left(a_{2}, y\right)\right] .
$$


So, considering for each $t, t \in\{1, \ldots, p\}, a_{1}=x_{t}$ and $a_{2}=-x_{t}^{*}$, we have the convexity of $\mathcal{R}_{\delta}$ if $x_{t} h_{\delta}\left(x_{t}, y\right)+x_{t}^{*} h_{\delta}\left(-x_{t}^{*}, y\right) \geq 0$. Thus, applying Jensen inequality, we obtain

$$
\begin{aligned}
& \prod_{t=1}^{p} E\left[F_{Z}\left(\frac{x_{t}}{\widetilde{U}_{t}^{\frac{1}{\delta}}}\right)-F_{Z}\left(-\frac{x_{t}^{*}}{\widetilde{U}_{t}^{\frac{1}{\delta}}}\right)\right] \prod_{t=p+1}^{n}\left[F_{Z}\left(\frac{x_{t}}{v_{t}^{\frac{1}{\delta}}}\right)-F_{Z}\left(-\frac{x_{t}^{*}}{v_{t}^{\frac{1}{\delta}}}\right)\right] \geq \\
& \quad \geq \prod_{t=1}^{p}\left[F_{Z}\left(\frac{x_{t}}{\left[E\left(\widetilde{U}_{t}\right)\right]^{\frac{1}{\delta}}}\right)-F_{Z}\left(-\frac{x_{t}^{*}}{\left[E\left(\widetilde{U}_{t}\right)\right]^{\frac{1}{\delta}}}\right)\right] \prod_{t=p+1}^{n}\left[F_{Z}\left(\frac{x_{t}}{v_{t}^{\frac{1}{\delta}}}\right)-F_{Z}\left(-\frac{x_{t}^{*}}{v_{t}^{\frac{1}{\delta}}}\right)\right] .
\end{aligned}
$$

As, under the hypotheses, $E\left(\widetilde{U}_{t}\right)$ exists and is equal to

$$
u_{t}=\sum_{i=1}^{t-1}\left[\alpha_{i}\left(x_{t-i}\right)^{\delta}+\beta_{i}\left(x_{t-i}^{*}\right)^{\delta}\right]+E\left(\sigma_{t}^{\delta}\right)\left[1-\sum_{i=1}^{t-1}\left(\alpha_{i} \phi_{1, \delta}+\beta_{i} \phi_{2, \delta}\right)\right],
$$

the inequality presented in point (a1) is then established.

(a2) The inequality corresponding to $q=0$ and $p>n \geq 1$ is analogously obtained.

In fact, as $\delta>0$, if $X_{t}^{+} \leq x_{t}$ and $X_{t}^{-} \leq x_{t}^{*}, t \in\{1, \ldots, n\}$, we obtain

- if $t \in\{2, \ldots, n\}, \sigma_{t}^{\delta} \leq \widetilde{U}_{t}$, as, for $i \in\{1, \ldots, t-1\}, 1 \leq t-i \leq n-1$ and, for $i \in\{t, \ldots, p\}, t-i \leq 0$;

- if $t=1, \sigma_{1}^{\delta}=\alpha_{0}+\sum_{i=1}^{p}\left[\alpha_{i}\left(X_{1-i}^{+}\right)^{\delta}+\beta_{i}\left(X_{1-i}^{-}\right)^{\delta}\right]$, and so $\sigma_{1}=\widetilde{U}_{1}$.

In consequence, we have the inequality

$$
F_{\left(X_{1}^{+}, X_{1}^{-}, \ldots, X_{n}^{+}, X_{n}^{-}\right)}\left(x_{1}, x_{1}^{*}, \ldots, x_{n}, x_{n}^{*}\right) \geq P\left(-\frac{x_{t}^{*}}{\widetilde{U}_{t}^{\frac{1}{\delta}}} \leq Z_{t} \leq \frac{x_{t}}{\widetilde{U}_{t}^{\frac{1}{\delta}}}, t=1, \ldots, n\right),
$$

from which the referred condition is similarly deduced.

b) The proof of this last inequality is also analogous to the previous ones. In fact, if $X_{t}^{+} \leq x_{t}$ and $X_{t}^{-} \leq x_{t}^{*}, t \in\{1, \ldots, n\}$, and introducing

$$
\widetilde{W}_{t}=\alpha_{0} \sum_{j=1}^{t-1} \gamma_{1}^{j-1}+\sum_{j=1}^{t-1} \gamma_{1}^{j-1}\left[\alpha_{1}\left(x_{t-j}\right)^{\delta}+\beta_{1}\left(x_{t-j}^{*}\right)^{\delta}\right]+\gamma_{1}^{t-1} \sigma_{1}^{\delta}
$$

we may write

$$
\text { - if } t \in\{2, \ldots, n\}, \sigma_{t}^{\delta} \leq \alpha_{0}+\alpha_{1}\left(x_{t-1}\right)^{\delta}+\beta_{1}\left(x_{t-1}^{*}\right)^{\delta}+\gamma_{1} \sigma_{t-1}^{\delta} \leq \widetilde{W}_{t},
$$


- if $t=1$, we have $\sigma_{1}=\widetilde{W}_{1}$,

and so

$$
F_{\left(X_{1}^{+}, X_{1}^{-}, \ldots, X_{n}^{+}, X_{n}^{-}\right)}\left(x_{1}, x_{1}^{*}, \ldots, x_{n}, x_{n}^{*}\right) \geq P\left(-\frac{x_{t}^{*}}{\widetilde{W}_{t}^{\frac{1}{\delta}}} \leq Z_{t} \leq \frac{x_{t}}{\widetilde{W}_{t}^{\frac{1}{\delta}}}, t=1, \ldots, n\right),
$$

with $\widetilde{W}_{t}^{\frac{1}{\delta}} \underline{X}_{0}$-measurable $(t=1, \ldots, n)$. Following the same steps the inequality in b) is obtained.

To illustrate this theorem, let us consider the cases $n=1$ and $n=2$ for the $\delta$-TGARCH(1,1) model.

When $n=1$, if $x_{1} h_{\delta}\left(x_{1}, y\right)+x_{1}^{*} h_{\delta}\left(-x_{1}^{*}, y\right) \geq 0$, for all $y \in\left[\theta^{\delta},+\infty[\right.$, then

$$
F_{\left(X_{1}^{+}, X_{1}^{-}\right)}\left(x_{1}, x_{1}^{*}\right) \geq F_{Z}\left(\frac{x_{1}}{\varpi_{1}}\right)-F_{Z}\left(-\frac{x_{1}^{*}}{\varpi_{1}}\right)
$$

considering the notation $\varpi_{1}=\left[E\left(\sigma_{t}^{\delta}\right)\right]^{1 / \delta}$.

When $n=2$, if, for $t=1,2$, we have $x_{t} h_{\delta}\left(x_{t}, y\right)+x_{t}^{*} h_{\delta}\left(-x_{t}^{*}, y\right) \geq 0$, for all $y \in\left[\theta^{\delta},+\infty[\right.$, then

$$
\begin{aligned}
& F_{\left(X_{1}^{+}, X_{1}^{-}, X_{2}^{+}, X_{2}^{-}\right)}\left(x_{1}, x_{1}^{*}, x_{2}, x_{2}^{*}\right) \geq \\
& \quad \geq\left[F_{Z}\left(\frac{x_{1}}{\varpi_{1}}\right)-F_{Z}\left(-\frac{x_{1}^{*}}{\varpi_{1}}\right)\right]\left[F_{Z}\left(\frac{x_{2}}{\varpi\left(x_{1}, x_{1}^{*}\right)}\right)-F_{Z}\left(-\frac{x_{2}^{*}}{\varpi\left(x_{1}, x_{1}^{*}\right)}\right)\right],
\end{aligned}
$$

with $\varpi_{1}=\left[E\left(\sigma_{t}^{\delta}\right)\right]^{1 / \delta}$ and $\varpi\left(x_{1}, x_{1}^{*}\right)=\left[\alpha_{0}+\alpha_{1}\left(x_{1}\right)^{\delta}+\beta_{1}\left(x_{1}^{*}\right)^{\delta}+\gamma_{1} E\left(\sigma_{t}^{\delta}\right)\right]^{1 / \delta}$.

\subsection{Bounds for the distribution function of $\left(X_{1}, \ldots, X_{n}\right)$}

We present now bounds for the distribution function of $\left(X_{1}, \ldots, X_{n}\right)$ considering the three following regions for $\left(x_{1}, \ldots, x_{n}\right) \in \mathbb{R}^{n}:\left[0,+\infty\left[^{n},\right]-\infty, 0\right]^{n} \backslash\{0, \ldots, 0\}$ and $\left.\mathbb{R}^{n} \backslash(]-\infty, 0\right]^{n} \cup\left[0,+\infty\left[^{n}\right)\right.$.

\subsubsection{Region $\left[0,+\infty\left[^{n}\right.\right.$}

Let $X$ be a $\delta$-TGARCH $(p, q)$ process with $\delta>0$. Then, for every $\left(x_{1}, \ldots, x_{n}\right) \in$ $\left[0,+\infty\left[^{n}, n \in \mathbb{N}\right.\right.$, it is easily concluded that

$$
F_{\left(X_{1}, \ldots, X_{n}\right)}\left(x_{1}, \ldots, x_{n}\right) \leq \prod_{t=1}^{n} F_{Z}\left(\frac{x_{t}}{\theta}\right) .
$$


For the lower bound, we begin by writing the distribution function as a sum of probabilities of sets where the variables are bounded, up or above, by zero like, for $n=2$,

$$
\begin{array}{r}
F_{\left(X_{1}, X_{2}\right)}\left(x_{1}, x_{2}\right)=P\left(X_{1}<0, X_{2}<0\right)+P\left(X_{1}<0,0 \leq X_{2} \leq x_{2}\right)+ \\
+P\left(0 \leq X_{1} \leq x_{1}, X_{2}<0\right)+P\left(0 \leq X_{1} \leq x_{1}, 0 \leq X_{2} \leq x_{2}\right) .
\end{array}
$$

In order to facilitate the reading, we introduce some notation and illustrate its use in the case $n=3$. As $\left.\left.]-\infty, x_{t}\right]=\right]-\infty, 0\left[\cup\left[0, x_{t}\right]\right.$, let us denote these intervals as $\left.C_{t}^{(-1)}=\right]-\infty, 0\left[\right.$ and $C_{t}^{(+1)}=\left[0, x_{t}\right]$. We note that the exponent used in this notation is also used as a number, that is, $C_{t}^{(-1)^{2}}=C_{t}^{(+1)}$ and $C_{t}^{(-1)^{3}}=C_{t}^{(-1)}$, for example. In this way, we have

$$
\begin{aligned}
\left.\left.\prod_{t=1}^{3}\right]-\infty, x_{t}\right]= & \prod_{t=1}^{3}\left(C_{t}^{(+1)} \cup C_{t}^{(-1)}\right) \\
= & C_{1}^{(+1)} \times C_{2}^{(+1)} \times C_{3}^{(+1)} \cup C_{1}^{(+1)} \times C_{2}^{(+1)} \times C_{3}^{(-1)} \cup \\
& \cup C_{1}^{(+1)} \times C_{2}^{(-1)} \times C_{3}^{(+1)} \cup C_{1}^{(+1)} \times C_{2}^{(-1)} \times C_{3}^{(-1)} \cup \\
& \cup C_{1}^{(-1)} \times C_{2}^{(+1)} \times C_{3}^{(+1)} \cup C_{1}^{(-1)} \times C_{2}^{(+1)} \times C_{3}^{(-1)} \cup \\
& \cup C_{1}^{(-1)} \times C_{2}^{(-1)} \times C_{3}^{(+1)} \cup C_{1}^{(-1)} \times C_{2}^{(-1)} \times C_{3}^{(-1)} .
\end{aligned}
$$

This Cartesian product is written as the union of $2^{3}$ Cartesian products, where the exponents are the arrangements with replacement of the numbers $(+1)$ and $(-1)$, three to three. For the condensed representation of this union, we consider the triplet $\left((-1)^{\left\lfloor\frac{k-1}{2^{2}}\right\rfloor},(-1)^{\left\lfloor\frac{k-1}{2^{1}}\right\rfloor},(-1)^{\left\lfloor\frac{k-1}{2^{0}}\right\rfloor}\right)$, where $\lfloor x\rfloor$ denotes the integer part of number $x$. With this triplet and with $k$ ranging from 1 to $2^{3}$, we recover the arrangements with replacement of the numbers $(+1)$ and $(-1)$, three to three, in the same order as that displayed. So,

$$
\begin{aligned}
& \left.\left.\prod_{t=1}^{3}\right]-\infty, x_{t}\right]=\bigcup_{k=1}^{2^{3}}\left(C_{1}^{(-1)^{\left\lfloor\frac{k-1}{2^{2}}\right\rfloor}} \times C_{2}^{(-1)^{\left\lfloor\frac{k-1}{2^{1}}\right\rfloor}} \times C_{3}^{(-1)^{\left\lfloor\frac{k-1}{2^{0}}\right\rfloor}}\right) \\
& =\bigcup_{k=1}^{2^{3}}\left(\prod_{t=1}^{3} C_{t}^{(-1)^{\left\lfloor\frac{k-1}{2^{3}-t}\right\rfloor}}\right) \text {. }
\end{aligned}
$$

The result concerning the lower bound can then be stated. 
Theorem 3. Let $X$ be a $\delta$-TGARCH$(p, q)$ process such that $Z_{t}$ are absolutely continuous variables. For every $\left(x_{1}, \ldots, x_{n}\right) \in\left[0,+\infty\left[^{n}, n \in \mathbb{N}\right.\right.$, we have

$$
\begin{aligned}
& F_{\left(X_{1}, \ldots, X_{n}\right)}\left(x_{1}, \ldots, x_{n}\right) \geq \\
& \geq\left[F_{Z}(0)\right]^{n}+\sum_{k=1}^{n} F_{\left(X_{1}^{+}, X_{1}^{-}, \ldots, X_{k}^{+}, X_{k}^{-}\right)}\left(x_{1}, 0, \ldots, x_{k}, 0\right)\left[F_{Z}(0)\right]^{n-k}+ \\
& \quad+\sum_{\substack{k=1 \\
k \neq 2^{0}, 2^{1}, \ldots, 2^{n}}}^{2^{n}} F_{\left(X_{1}^{+}, X_{1}^{-}, \ldots, X_{n}^{+}, X_{n}^{-}\right)}\left(a_{k, 1}, a_{k, 1}^{*}, \ldots, a_{k, n}, a_{k, n}^{*}\right),
\end{aligned}
$$

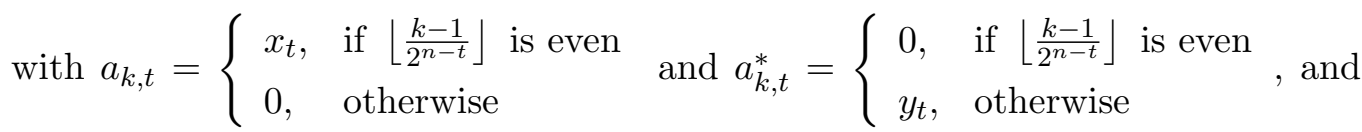
$y_{t}$ arbitrarily fixed in $[0,+\infty[$.

Proof. Let $\left(x_{1}, \ldots, x_{n}\right) \in\left[0,+\infty\left[{ }^{n}\right.\right.$. Using the procedure previously referred, the following equality holds for the set $\left.\left.\prod_{t=1}^{n}\right]-\infty, x_{t}\right]$ :

$$
\left.\left.\prod_{t=1}^{n}\right]-\infty, x_{t}\right]=\prod_{t=1}^{n}\left(C_{t}^{(+1)} \cup C_{t}^{(-1)}\right)=\bigcup_{k=1}^{2^{n}}\left(\prod_{t=1}^{n} C_{t}^{(-1)^{\left\lfloor\frac{k-1}{2^{n-t}}\right\rfloor}}\right) .
$$

As $C_{t}^{(+1)} \cap C_{t}^{(-1)}=\varnothing, t=1, \ldots, n$, then

$$
\prod_{t=1}^{n} C_{t}^{(-1)^{\left\lfloor\frac{k_{1}-1}{2^{n-t}}\right\rfloor}} \cap \prod_{t=1}^{n} C_{t}^{(-1)^{\left\lfloor\frac{k_{2}-1}{2^{n-t}}\right\rfloor}}=\varnothing
$$

for $k_{1}, k_{2} \in\left\{1, \ldots, 2^{n}\right\}$ such that $k_{1} \neq k_{2}$. So,

$$
F_{\left(X_{1}, \ldots, X_{n}\right)}\left(x_{1}, \ldots, x_{n}\right)=\sum_{k=1}^{2^{n}} P\left(\left(X_{1}, \ldots, X_{n}\right) \in \prod_{t=1}^{n} C_{t}^{(-1)\left\lfloor\frac{k-1}{2^{n-t}}\right\rfloor}\right) .
$$

Let us evaluate now $P\left(\left(X_{1}, \ldots, X_{n}\right) \in \prod_{t=1}^{n} C_{t}^{(-1)^{\left\lfloor\frac{k-1}{2^{n-t}}\right\rfloor}}\right)$, for $k=2^{n-j}$, with $j$ ranging from 0 to $n$. obtain

Taking into account that for $k=2^{0}$ we have $\prod_{t=1}^{n} C_{t}^{(-1)^{\left\lfloor\frac{k-1}{2^{n-t}}\right\rfloor}}=\prod_{t=1}^{n} C_{t}^{(+1)}$, we

$$
\begin{aligned}
P\left(\left(X_{1}, \ldots, X_{n}\right) \in \prod_{t=1}^{n} C_{t}^{(+1)}\right) & =P\left(0 \leq X_{t} \leq x_{t}, t=1, \ldots, n\right) \\
& =P\left(X_{t}^{+} \leq x_{t}, X_{t}^{-} \leq 0, t=1, \ldots, n\right) \\
& =F_{\left(X_{1}^{+}, X_{1}^{-}, \ldots, X_{n}^{+}, X_{n}^{-}\right)}\left(x_{1}, 0, \ldots, x_{n}, 0\right) .
\end{aligned}
$$


Taking $k=2^{n}$, we have $\prod_{t=1}^{n} C_{t}^{(-1)^{\left\lfloor\frac{k-1}{2^{n-t}}\right\rfloor}}=\prod_{t=1}^{n} C_{t}^{(-1)}$, and so

$$
\begin{aligned}
P\left(\left(X_{1}, \ldots, X_{n}\right) \in \prod_{t=1}^{n} C_{t}^{(-1)}\right) & =P\left(X_{t}<0, t=1, \ldots, n\right) \\
& =P\left(Z_{t}<0, t=1, \ldots, n\right) \\
& =\left[F_{Z}(0)\right]^{n},
\end{aligned}
$$

since $\sigma_{t}>0$, for $t \in\{1, \ldots, n\}$, and $Z_{1}, \ldots, Z_{n}$ are i.i.d. absolutely continuous variables.

Finally, let us analyze the case $k=2^{n-j}$, with $j \in\{1, \ldots, n-1\}$. In this case $\prod_{t=1}^{n} C_{t}^{(-1)^{\left\lfloor\frac{k-1}{2^{n-t}}\right\rfloor}}=\prod_{t=1}^{j} C_{t}^{(+1)} \times \prod_{t=j+1}^{n} C_{t}^{(-1)}$, and so

$$
\begin{aligned}
& P\left(\left(X_{1}, \ldots, X_{n}\right) \in \prod_{t=1}^{j} C_{t}^{(+1)} \times \prod_{t=j+1}^{n} C_{t}^{(-1)}\right)= \\
& \quad=P\left(0 \leq X_{t} \leq x_{t}, t=1, \ldots, j, X_{t}<0, t=j+1, \ldots, n\right) \\
& \quad=F_{\left(X_{1}^{+}, X_{1}^{-}, \ldots, X_{j}^{+}, X_{j}^{-}\right)}\left(x_{1}, 0, \ldots, x_{j}, 0\right) \times\left[F_{Z}(0)\right]^{n-j}
\end{aligned}
$$

taking into account that $Z_{t}$ is independent of $\underline{X}_{t-1}$.

For the remaining values of $k$, that is, for $k \in\left\{1, \ldots, 2^{n}\right\}$ such that $k \neq 2^{n-j}$ with $j$ ranging from 0 to $n$, we obtain now a lower bound for $P\left(\left(X_{1}, \ldots, X_{n}\right) \in \prod_{t=1}^{n} C_{t}^{(-1)\left\lfloor\frac{k-1}{\left.2^{n-t}\right\rfloor}\right.}\right)$.

Let us fix arbitrarily $\left(y_{1}, \ldots, y_{n}\right)$ in $] 0,+\infty\left[^{n}\right.$.

For $t=1, \ldots, n$, we have $\left[-y_{t}, 0\left[\subset C_{t}^{(-1)}\right.\right.$. Denoting $D_{t}^{(-1)}=\left[-y_{t}, 0[\right.$ and $D_{t}^{(+1)}=C_{t}^{(+1)}$, we get

$$
P\left(\left(X_{1}, \ldots, X_{n}\right) \in \prod_{t=1}^{n} C_{t}^{(-1)^{\left\lfloor\frac{k-1}{2^{n-t}}\right\rfloor}}\right) \geq P\left(\left(X_{1}, \ldots, X_{n}\right) \in \prod_{t=1}^{n} D_{t}^{(-1)^{\left\lfloor\frac{k-1}{2^{n-t}}\right\rfloor}}\right) .
$$

Considering now

$$
a_{k, t}=\left\{\begin{array}{ll}
x_{t}, & \text { if }\left\lfloor\frac{k-1}{\left.2^{n-t}\right\rfloor}\right. \text { is even } \\
0, & \text { otherwise }
\end{array} \quad \text { and } a_{k, t}^{*}=\left\{\begin{array}{ll}
0, & \text { if }\left\lfloor\frac{k-1}{\left.2^{n-t}\right\rfloor}\right. \text { is even } \\
y_{t}, & \text { otherwise }
\end{array},\right.\right.
$$

we obtain, if $\left\lfloor\frac{k-1}{2^{n-t}}\right\rfloor$ is even, $\left[-a_{k, t}^{*}, a_{k, t}\right]=\left[0, x_{t}\right]=D_{t}^{(+1)}$ and, if $\left\lfloor\frac{k-1}{2^{n-t}}\right\rfloor$ is odd, 


$$
\begin{aligned}
& {\left[-a_{k, t}^{*}, a_{k, t}\right]=\left[-y_{t}, 0\right]=D_{t}^{(-1)} \cup\{0\} \text {. So, }} \\
& \begin{aligned}
P\left(\left(X_{1}, \ldots, X_{n}\right) \in \prod_{t=1}^{n} D_{t}^{\left.(-1)^{\frac{k-1}{2^{n-t}}}\right]}\right) & =P\left(\left(X_{1}, \ldots, X_{n}\right) \in \prod_{t=1}^{n}\left[-a_{k, t}^{*}, a_{k, t}\right]\right) \\
& =P\left(X_{t}^{+} \leq a_{k, t}, X_{t}^{-} \leq a_{k, t}^{*}, t=1, \ldots, n\right) \\
& =F_{\left(X_{1}^{+}, X_{1}^{-}, \ldots, X_{n}^{+}, X_{n}^{-}\right)}\left(a_{k, 1}, a_{k, 1}^{*}, \ldots, a_{k, n}, a_{k, n}^{*}\right),
\end{aligned}
\end{aligned}
$$

as $\left(Z_{t}\right)$, and consequently $\left(X_{t}\right)$, are absolutely continuous random variables.

The conclusions obtained for all the values of $k$ from 1 to $2^{n}$, give the stated lower bound.

We note that this theorem is valid for any value of $\delta$, positive or negative.

Let us now exemplify theorem 3 , starting with $n=1$. In this case, for $x_{1} \geq 0$, $F_{X_{1}}\left(x_{1}\right) \geq F_{Z}(0)+F_{\left(X_{1}^{+}, X_{1}^{-}\right)}\left(x_{1}, 0\right)$ and, if we then apply theorem 2 to the second term of the sum, we get the bound obtained in case (a1) of theorem 1 with exactly the same conditions regarding the function $h_{\delta}\left(x_{1}, y\right)$.

When $n=2$, for $\left(x_{1}, x_{2}\right) \in\left[0,+\infty\left[^{2}\right.\right.$,

$$
\begin{aligned}
F_{\left(X_{1}, X_{2}\right)}\left(x_{1}, x_{2}\right) \geq & {\left[F_{Z}(0)\right]^{2}+F_{\left(X_{1}^{+}, X_{1}^{-}\right)}\left(x_{1}, 0\right)\left[F_{Z}(0)\right]+} \\
& +F_{\left(X_{1}^{+}, X_{1}^{-}, X_{2}^{+}, X_{2}^{-}\right)}\left(x_{1}, 0, x_{2}, 0\right)+F_{\left(X_{1}^{+}, X_{1}^{-}, X_{2}^{+}, X_{2}^{-}\right)}\left(0, y_{1}, x_{2}, 0\right),
\end{aligned}
$$

as $a_{3,1}=0, a_{3,1}^{*}=y_{1}, a_{3,2}=x_{2}$ and $a_{3,2}^{*}=0$, with this bound valid for any $y_{1}$ but the quality of the bound improves as the value of $y_{1}$ increases. This result, as in the case $n=1$, is only usable if now combined with theorem 2 , so it is important to refer that the hypotheses of theorem 2 imply all the hypotheses of theorem 3 and one consequence is that $y_{1}$ should be chosen as the greatest positive real such that $h_{\delta}\left(-y_{1}, y\right) \geq 0$, for all $y \in\left[\theta^{\delta},+\infty[\right.$.

\subsubsection{Region $]-\infty, 0]^{n} \backslash\{(0, \ldots, 0)\}$}

In the region $]-\infty, 0]^{n} \backslash\{(0, \ldots, 0)\}$, the lower bound is also a natural generalization of the theorem related to the marginal distribution, that is,

$$
F_{\left(X_{1}, \ldots, X_{n}\right)}\left(x_{1}, \ldots, x_{n}\right) \geq \prod_{t=1}^{n} F_{Z}\left(\frac{x_{t}}{\theta}\right) .
$$

In what concerns the upper bound for the distribution function in this region, the following result is stated using again the distribution function of the vector $\left(X_{1}^{+}, X_{1}^{-}, \ldots, X_{n}^{+}, X_{n}^{-}\right)$and it does not depend on the sign of $\delta$. 
Theorem 4. Let $X$ be a $\delta$-TGARCH$(p, q)$ process such that $Z_{t}$ are absolutely continuous variables. For every $\left.\left.\left(x_{1}, \ldots, x_{n}\right) \in\right]-\infty, 0\right]^{n} \backslash\{(0, \ldots, 0)\}, n \in \mathbb{N}$,

$$
F_{\left(X_{1}, \ldots, X_{n}\right)}\left(x_{1}, \ldots, x_{n}\right) \leq\left[F_{Z}(0)\right]^{n}-F_{\left(X_{1}^{+}, X_{1}^{-}, \ldots, X_{n}^{+}, X_{n}^{-}\right)}\left(0,-x_{1}, \ldots, 0,-x_{n}\right) .
$$

Proof. The beginning of the proof is analogous to that of theorem 3, starting with the set $]-\infty, 0]^{n}$.

Let $\left.\left.\left(x_{1}, \ldots, x_{n}\right) \in\right]-\infty, 0\right]^{n} \backslash\{(0, \ldots, 0)\}$. Since

$$
]-\infty, 0]^{n}=\prod_{t=1}^{n}\left(\mathcal{C}_{t}^{(+1)} \cup \mathcal{C}_{t}^{(-1)}\right)=\bigcup_{k=1}^{2^{n}}\left(\prod_{t=1}^{n} \mathcal{C}_{t}^{(-1)^{\left\lfloor\frac{k-1}{2^{n-t}}\right\rfloor}}\right),
$$

where, for $t=1, \ldots, n$, we have $\left.\mathcal{C}_{t}^{(-1)}=\right]-\infty, x_{t}\left[\right.$ and $\mathcal{C}_{t}^{(+1)}=\left[x_{t}, 0\right]$, and so $\mathcal{C}_{t}^{(+1)} \cap \mathcal{C}_{t}^{(-1)}=\varnothing$, then, $F_{\left(X_{1}, \ldots, X_{n}\right)}(0, \ldots, 0)=\left[F_{Z}(0)\right]^{n}$ is also equal to

$$
F_{\left(X_{1}, \ldots, X_{n}\right)}(0, \ldots, 0)=\sum_{k=1}^{2^{n}} P\left(\left(X_{1}, \ldots, X_{n}\right) \in \prod_{t=1}^{n} \mathcal{C}_{t}^{(-1)^{\left\lfloor\frac{k-1}{2^{n-t}}\right\rfloor}}\right) .
$$

As for $k=2^{n}, \prod_{t=1}^{n} C_{t}^{(-1)^{\left\lfloor\frac{k-1}{2^{n-t}}\right\rfloor}}=\prod_{t=1}^{n} C_{t}^{(-1)}$, the last term of this sum is $F_{\left(X_{1}, \ldots, X_{n}\right)}(0, \ldots, 0)=\left[F_{Z}(0)\right]^{n}$.

Moreover, the first one, corresponding to $k=1$, is such that $\prod_{t=1}^{n} \mathcal{C}_{t}^{(-1)^{\left\lfloor\frac{k-1}{2^{n-t}}\right\rfloor}}=$ $\prod_{t=1}^{n} \mathcal{C}_{t}^{(+1)}$, and so

$$
\begin{aligned}
P\left(\left(X_{1}, \ldots, X_{n}\right) \in \prod_{t=1}^{n} \mathcal{C}_{t}^{(+1)}\right) & =P\left(0 \leq X_{t} \leq-x_{t}, t=1, \ldots, n\right) \\
& =F_{\left(X_{1}^{+}, X_{1}^{-}, \ldots, X_{n}^{+}, X_{n}^{-}\right)}\left(0,-x_{1}, \ldots, 0,-x_{n}\right),
\end{aligned}
$$

Consequently,

$$
\begin{aligned}
F_{\left(X_{1}, \ldots, X_{n}\right)}\left(x_{1}, \ldots, x_{n}\right)= & {\left[F_{Z}(0)\right]^{n}-F_{\left(X_{1}^{+}, X_{1}^{-}, \ldots, X_{n}^{+}, X_{n}^{-}\right)}\left(0,-x_{1}, \ldots, 0,-x_{n}\right)+} \\
& -\sum_{k=2}^{2^{n-1}} P\left(\left(X_{1}, \ldots, X_{n}\right) \in \prod_{t=1}^{n} \mathcal{C}_{t}^{\left.(-1)^{\left\lfloor\frac{k-1}{2^{n-t}}\right\rfloor}\right)}\right.
\end{aligned}
$$

from which we conclude.

As in the previous theorems, in the following we illustrate theorem 4, starting with $n=1$. In this case, for $x_{1}<0, F_{X_{1}}\left(x_{1}\right) \leq F_{Z}(0)-F_{\left(X_{1}^{+}, X_{1}^{-}\right)}\left(0,-x_{1}\right)$ and, 
if we then apply theorem 2, we get the bound obtained in case (b1) of theorem 1 with exactly the same conditions regarding the function $h_{\delta}\left(x_{1}, y\right)$.

When $n=2$, for $\left.\left.\left(x_{1}, x_{2}\right) \in\right]-\infty, 0\right]^{2} \backslash\{(0,0)\}$,

$$
F_{\left(X_{1}, X_{2}\right)}\left(x_{1}, x_{2}\right) \leq\left[F_{Z}(0)\right]^{2}-F_{\left(X_{1}^{+}, X_{1}^{-}, X_{2}^{+}, X_{2}^{-}\right)}\left(0,-x_{1}, 0,-x_{2}\right) .
$$

and, again, to use this result in practice it has to be combined with theorem 2 .

\subsubsection{Region $\left.\mathrm{R}^{n} \backslash(]-\infty, 0\right]^{n} \cup\left[0,+\infty\left[^{n}\right)\right.$}

In the region $\left.R^{n} \backslash(]-\infty, 0\right]^{n} \cup\left[0,+\infty\left[^{n}\right)\right.$, with $n \in \mathbb{N} \backslash\{1\}$, we consider only the $\delta$-TGARCH model with positive power. We use the following sets of indices:

$$
\begin{aligned}
& \mathcal{I}^{+}=\left\{t \in\{1, \ldots, n\}: x_{t}>0\right\}, \text { with } \breve{\eta}=\min \mathcal{I}^{+} \text {and } \# \mathcal{I}^{+}=k ; \\
& \mathcal{I}^{-}=\left\{t \in\{1, \ldots, n\}: x_{t}<0\right\}, \text { with } \ddot{\eta}=\min \mathcal{I}^{-} \text {and } \# \mathcal{I}^{-}=\kappa ; \\
& \mathcal{I}^{+*}=\left\{t \in \mathcal{I}^{+}: t>\ddot{\eta}\right\} \text { such that } \# \mathcal{I}^{+*}=k^{*} ; \\
& \mathcal{I}^{-*}=\left\{t \in \mathcal{I}^{-}: t>\breve{\eta}\right\} \text { such that } \# \mathcal{I}^{-*}=\kappa^{*} .
\end{aligned}
$$

The following theorem states an upper bound for the distribution function in study.

Theorem 5. Let $X$ be a $\delta$-TGARCH$(p, q)$ process such that $\delta>0$. Let $\left(x_{1}, \ldots, x_{n}\right)$ be any element of $\left.\mathbb{R}^{n} \backslash(]-\infty, 0\right]^{n} \cup\left[0,+\infty\left[^{n}\right)\right.$, for $n \in \mathbb{N} \backslash\{1\}$, with $k$ positive coordinates, for $1 \leq k<n$. Then,

a) if $x_{1} \leq 0$, we have

$$
\begin{aligned}
& \quad F_{\left(X_{1}, \ldots, X_{n}\right)}\left(x_{1}, \ldots, x_{n}\right) \leq \\
& \quad \leq F_{\left(X_{1}, \ldots, X_{\breve{\eta}-1}\right)}\left(x_{1}, \ldots, x_{\breve{\eta}-1}\right) \prod_{t \in \mathcal{I}^{+}} F_{Z}\left(\frac{x_{t}}{\theta}\right) \times\left[F_{Z}(0)\right]^{n-k-(\breve{\eta}-1)},
\end{aligned}
$$

b) if $x_{1} \geq 0$, we have

$$
\begin{aligned}
& F_{\left(X_{1}, \ldots, X_{n}\right)}\left(x_{1}, \ldots, x_{n}\right) \leq \\
& \quad \leq F_{\left(X_{1}, \ldots, X_{\ddot{\eta}-1}\right)}\left(x_{1}, \ldots, x_{\ddot{\eta}-1}\right) \prod_{t \in \mathcal{I}^{+*}} F_{Z}\left(\frac{x_{t}}{\theta}\right) \times\left[F_{Z}(0)\right]^{n-k^{*}-(\ddot{\eta}-1)} .
\end{aligned}
$$

Proof. We present only the proof of part a), as the other is analogous.

Let $\left.\left(x_{1}, \ldots, x_{n}\right) \in \mathbb{R}^{n} \backslash(]-\infty, 0\right]^{n} \cup\left[0,+\infty\left[^{n}\right)\right.$, with $n$ fixed in $\mathbb{N} \backslash\{1\}$, such that $k$ of its coordinates are positive, with $1 \leq k<n$. 
If $x_{1} \leq 0$, we get

$$
\begin{aligned}
F_{\left(X_{1}, \ldots, X_{n}\right)}\left(x_{1}, \ldots, x_{n}\right) & =P\left(X_{1} \leq x_{1}, \ldots, X_{\breve{\eta}-1} \leq x_{\breve{\eta}-1}, X_{\breve{\eta}} \leq x_{\breve{\eta}}, \ldots, X_{n} \leq x_{n}\right) \\
& =P\left(X_{1} \leq x_{1}, \ldots, X_{\breve{\eta}-1} \leq x_{\breve{\eta}-1}, Z_{\breve{\eta}} \leq \frac{x_{\breve{\eta}}}{\sigma_{\breve{\eta}}}, \ldots, Z_{n} \leq \frac{x_{n}}{\sigma_{n}}\right)
\end{aligned}
$$

with $\breve{\eta} \geq 2$. So, for $1 \leq t \leq \breve{\eta}-1, x_{t} \leq 0$ and, for $t=\breve{\eta}, x_{t}>0$.

For $\breve{\eta} \leq t \leq n$, we upper-bound each coordinate in the following way:

i) if $x_{t} \leq 0$, then, as $\sigma_{t}>0$, we have $\frac{x_{t}}{\sigma_{t}} \leq 0$ and so $\left\{Z_{t} \leq \frac{x_{t}}{\sigma_{t}}\right\} \subseteq\left\{Z_{t} \leq 0\right\}$;

ii) if $x_{t}>0$, as $\sigma_{t} \geq \theta$, then $\frac{x_{t}}{\sigma_{t}} \leq \frac{x_{t}}{\theta}$ and so, $\left\{Z_{t} \leq \frac{x_{t}}{\sigma_{t}}\right\} \subseteq\left\{Z_{t} \leq \frac{x_{t}}{\theta}\right\}$.

Consequently

$$
F_{\left(X_{1}, \ldots, X_{n}\right)}\left(x_{1}, \ldots, x_{n}\right) \leq P\left(X_{1} \leq x_{1}, \ldots, X_{\breve{\eta}-1} \leq x_{\breve{\eta}-1}, Z_{\breve{\eta}} \leq u_{\breve{\eta}}, \ldots, Z_{n} \leq u_{n}\right),
$$

where $u_{t}=\left\{\begin{array}{cc}0, & \text { if } x_{t} \leq 0 \\ \frac{x_{t}}{\theta}, & \text { if } x_{t}>0\end{array}\right.$, for $t=\breve{\eta}, \ldots, n$, is non random. As $Z_{\breve{\eta}}, \ldots, Z_{n}$ are independent variables and independent of $\underline{X}_{\breve{\eta}-1}$, then

$$
\begin{aligned}
& P\left(X_{1} \leq x_{1}, \ldots, X_{\breve{\eta}-1} \leq x_{\breve{\eta}-1}, Z_{\breve{\eta}} \leq u_{\breve{\eta}}, \ldots, Z_{n} \leq u_{n}\right) \\
& \quad=F_{\left(X_{1}, \ldots, X_{\breve{\eta}-1}\right)}\left(x_{1}, \ldots, x_{\breve{\eta}-1}\right) \times F_{Z}\left(u_{\breve{\eta}}\right) \times \ldots \times F_{Z}\left(u_{n}\right) .
\end{aligned}
$$

Taking into account the $u_{t}$ definition, we conclude that

$$
\begin{aligned}
& F_{\left(X_{1}, \ldots, X_{n}\right)}\left(x_{1}, \ldots, x_{n}\right) \leq \\
& \quad \leq F_{\left(X_{1}, \ldots, X_{\breve{\eta}-1}\right)}\left(x_{1}, \ldots, x_{\breve{\eta}-1}\right) \prod_{t \in \mathcal{I}^{+}} F_{Z}\left(\frac{x_{t}}{\theta}\right) \times\left[F_{Z}(0)\right]^{n-k-(\breve{\eta}-1)} .
\end{aligned}
$$

We point out that, in case a), the dependence of the upper bound on the distribution function of $\left(X_{1}, \ldots, X_{t}\right)$, with $t<\breve{\eta}-1$, is addressed taking into consideration the previous studies since all the components of the point $\left(x_{1}, \ldots, x_{\breve{\eta}-1}\right)$ where this function is evaluated are non positives which enables the use of the results stated for the region $]-\infty, 0]^{n} \backslash\{(0, \ldots, 0)\}$. In case $\mathbf{b}$ ), an analogous situation occurs with points belonging now to $\left[0,+\infty\left[^{n}\right.\right.$.

In the next theorem we complete the bound of the distribution function. The proof is omitted due to its similarity with that of the previous theorem. 
Theorem 6. Let $X$ be a $\delta$-TGARCH$(p, q)$ process such that $\delta>0$. Let $\left(x_{1}, \ldots, x_{n}\right)$ be any point in $\left.\mathbb{R}^{n} \backslash(]-\infty, 0\right]^{n} \cup\left[0,+\infty\left[^{n}\right)\right.$, for $n \in \mathbb{N} \backslash\{1\}$, with $\kappa$ negative coordinates, for $1 \leq \kappa<n$. Then,

a) if $x_{1} \leq 0$, we have

$$
\begin{aligned}
& F_{\left(X_{1}, \ldots, X_{n}\right)}\left(x_{1}, \ldots, x_{n}\right) \geq \\
& \quad \geq F_{\left(X_{1}, \ldots, X_{\breve{\eta}-1}\right)}\left(x_{1}, \ldots, x_{\breve{\eta}-1}\right) \prod_{t \in \mathcal{I}^{-*}} F_{Z}\left(\frac{x_{t}}{\theta}\right) \times\left[F_{Z}(0)\right]^{n-\kappa^{*}-(\breve{\eta}-1)},
\end{aligned}
$$

b) if $x_{1} \geq 0$, we have

$$
\begin{aligned}
& F_{\left(X_{1}, \ldots, X_{n}\right)}\left(x_{1}, \ldots, x_{n}\right) \geq \\
& \quad \geq F_{\left(X_{1}, \ldots, X_{\ddot{\eta}-1}\right)}\left(x_{1}, \ldots, x_{\ddot{\eta}-1}\right) \prod_{t \in \mathcal{I}^{-}} F_{Z}\left(\frac{x_{t}}{\theta}\right) \times\left[F_{Z}(0)\right]^{n-\kappa-(\ddot{\eta}-1)} .
\end{aligned}
$$

We now exemplify theorems 5 and 6 when $n=2$.

- For $x_{1} \leq 0$ and $x_{2} \geq 0$, we have $\mathcal{I}^{+}=\{2\}, \breve{\eta}=2, k=1, \mathcal{I}^{-}=\{1\}, \ddot{\eta}=1$, $\kappa=1, \mathcal{I}^{+*}=\{2\}, k^{*}=1, \mathcal{I}^{-*}=\varnothing$ and $\kappa^{*}=0$, thus

$$
F_{X_{1}}\left(x_{1}\right) F_{Z}(0) \leq F_{\left(X_{1}, X_{2}\right)}\left(x_{1}, x_{2}\right) \leq F_{X_{1}}\left(x_{1}\right) F_{Z}\left(\frac{x_{2}}{\theta}\right) \text {. }
$$

- For $x_{1} \geq 0$ and $x_{2} \leq 0$, we have $\mathcal{I}^{+}=\{1\}, \breve{\eta}=1, k=1, \mathcal{I}^{-}=\{2\}, \ddot{\eta}=2$, $\kappa=1, \mathcal{I}^{+*}=\varnothing, k^{*}=0, \mathcal{I}^{-*}=\{2\}$ and $\kappa^{*}=1$, thus

$$
F_{X_{1}}\left(x_{1}\right) F_{Z}\left(\frac{x_{2}}{\theta}\right) \leq F_{\left(X_{1}, X_{2}\right)}\left(x_{1}, x_{2}\right) \leq F_{X_{1}}\left(x_{1}\right) F_{Z}(0) .
$$

These results now have to be combined with the ones obtained for the marginal distribution.

\section{Simulation study}

The theoretical bounds for the finite dimensional distributions of a process $X$ following a $\delta$-TGARCH model are now evaluated by means of a simulation study. This study is devoted to the bounds obtained for $F_{\left(X_{1}, X_{2}\right)}$ as in this case we are able to graphically compare the results, like we have done in the marginal distributions.

We consider the $\delta$-TGARCH $(1,1)$ model and we firstly resume the expressions of the bounds obtained for $n=2$. So, for each $\left(x_{1}, x_{2}\right)$, we have an upper and a 
lower bound for $F_{\left(X_{1}, X_{2}\right)}\left(x_{1}, x_{2}\right)$, denoted by $L S\left(x_{1}, x_{2}\right)$ and $L I\left(x_{1}, x_{2}\right)$, respectively. In order to facilitate the presentation, we consider $\mathbb{R}^{2}$ divided in its four quadrants, including the points $\left(0, x_{2}\right)$ and $\left(x_{1}, 0\right)$ in the adequate odd quadrant. Denoting $F_{Z}(0)$ by $a$ and considering $\theta=\left(\frac{\alpha_{0}}{1-\gamma_{1}}\right)^{1 / \delta}, \varpi_{1}=\left[E\left(\sigma_{t}^{\delta}\right)\right]^{1 / \delta}, \varpi_{2}(x)=$ $\left[\alpha_{0}+\alpha_{1} x^{\delta}+\gamma_{1} E\left(\sigma_{t}^{\delta}\right)\right]^{1 / \delta}$ and $\varpi_{3}(x)=\left[\alpha_{0}+\beta_{1} x^{\delta}+\gamma_{1} E\left(\sigma_{t}^{\delta}\right)\right]^{1 / \delta}$ with $y_{1}$ chosen as the greatest positive real such that $h_{\delta}\left(-y_{1}, y\right) \geq 0$, for all $y \in\left[\theta^{\delta},+\infty[\right.$, we get

$$
L S\left(x_{1}, x_{2}\right)= \begin{cases}F_{Z}\left(\frac{x_{1}}{\theta}\right) F_{Z}\left(\frac{x_{2}}{\theta}\right), & \left(x_{1}, x_{2}\right) \in 1 .^{o} Q \\ F_{Z}\left(\frac{x_{1}}{\varpi_{1}}\right) F_{Z}\left(\frac{x_{2}}{\theta}\right), & \left(x_{1}, x_{2}\right) \in 2 .^{o} Q \text { such that } \\ a^{2}-\left[a-F_{Z}\left(\frac{x_{1}}{\varpi_{1}}\right)\right] \times & h_{\delta}\left(x_{1}, y\right) \geq 0 \\ \times\left[a-F_{Z}\left(\frac{x_{2}}{\varpi_{3}\left(-x_{1}\right)}\right)\right], & \left(x_{1}, x_{2}\right) \in 3 .^{o} Q \text { such that } \\ F_{Z}\left(\frac{x_{1}}{\theta}\right) a, & \left(x_{1}, x_{2}\right) \in 4 .^{o} Q\end{cases}
$$

and $L I\left(x_{1}, x_{2}\right)=$

$$
= \begin{cases}F_{Z}\left(\frac{x_{1}}{\varpi_{1}}\right) a+ & \\ +\left[F_{Z}\left(\frac{x_{1}}{\varpi_{1}}\right)-a\right]\left[F_{Z}\left(\frac{x_{2}}{\varpi_{2}\left(x_{1}\right)}\right)-a\right]+ & \left(x_{1}, x_{2}\right) \in 1 .{ }^{o} Q \text { such that } \\ +\left[a-F_{Z}\left(-\frac{y_{1}}{\varpi_{1}}\right)\right]\left[F_{Z}\left(\frac{x_{2}}{\varpi_{3}\left(y_{1}\right)}\right)-a\right], & h_{\delta}\left(x_{1}, y\right) \geq 0 \text { and } h_{\delta}\left(x_{2}, y\right) \geq 0 \\ F_{Z}\left(\frac{x_{1}}{\theta}\right) a, & \left(x_{1}, x_{2}\right) \in 2 .{ }^{o} Q \\ F_{Z}\left(\frac{x_{1}}{\theta}\right) F_{Z}\left(\frac{x_{2}}{\theta}\right), & \left(x_{1}, x_{2}\right) \in 3 .^{o} Q \\ F_{Z}\left(\frac{x_{1}}{\varpi_{1}}\right) F_{Z}\left(\frac{x_{2}}{\theta}\right), & \left(x_{1}, x_{2}\right) \in 4 .^{o} Q \text { such that } \\ & h_{\delta}\left(x_{1}, y\right) \geq 0\end{cases}
$$

In the sequel of the simulation study done for the marginal distribution of $X$ when the marginal distribution of the generator process is Gaussian we consider the $\delta$-TGARCH $(1,1)$ model, with $\alpha_{0}=10, \alpha_{1}=0.3, \beta_{1}=0.5, \gamma_{1}=0.2$, and $\delta$ equal to $\frac{1}{2}$ (Figure 5 ) and 2 (Figure 6 ). In each figure, the same graphic is presented under four perspectives, with $L S\left(x_{1}, x_{2}\right)$ in orange, $L I\left(x_{1}, x_{2}\right)$ in blue and, in green, the empirical estimate of $F_{\left(X_{1}, X_{2}\right)}\left(x_{1}, x_{2}\right)$, calculated from 10000 realizations of the $X$ process. For readability, we only present the region $\left(x_{1}, x_{2}\right) \in$ $[-\theta \sqrt{1+\delta}, \theta \sqrt{1+\delta}]^{2}$, where it is assured that $h_{\delta}\left(x_{1}, y\right) \geq 0$ and $h_{\delta}\left(x_{2}, y\right) \geq 0$. 
Nevertheless, we point out that the theoretical bounds for $F_{\left(X_{1}, X_{2}\right)}\left(x_{1}, x_{2}\right)$ by means of $L S\left(x_{1}, x_{2}\right)$ and $L I\left(x_{1}, x_{2}\right)$ are valid in a larger region, as we easily see from their expressions. We considered $y_{1}=\theta \sqrt{1+\delta}$.

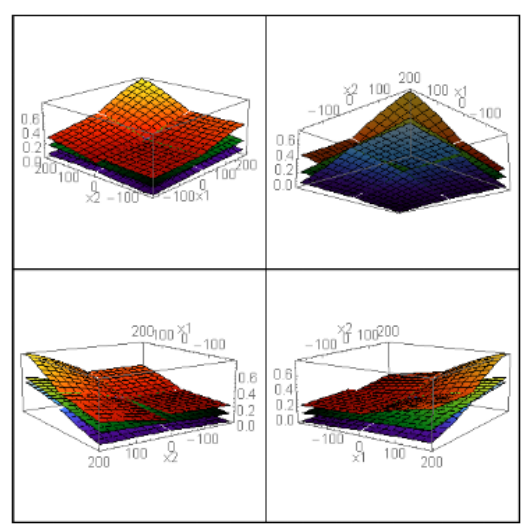

Figure 5: Graphical representation of $L S\left(x_{1}, x_{2}\right)$ (orange), $L I\left(x_{1}, x_{2}\right)$ (blue) and estimate of $F_{\left(X_{1}, X_{2}\right)}\left(x_{1}, x_{2}\right)$ (green), with $X \sim \frac{1}{2}$-TGARCH(1, 1$)$ and $Z_{t} \sim N(0,1)$

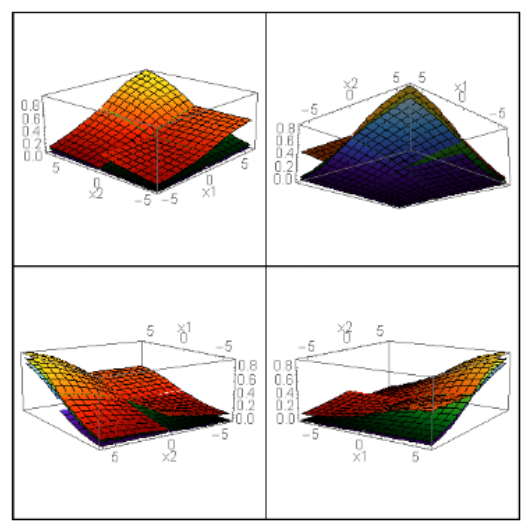

Figure 6: Graphical representation of $L S\left(x_{1}, x_{2}\right)$ (orange), $L I\left(x_{1}, x_{2}\right)$ (blue) and estimate of $F_{\left(X_{1}, X_{2}\right)}\left(x_{1}, x_{2}\right)$ (green), with $X \sim 2$-TGARCH $(1,1)$ and $Z_{t} \sim N(0,1)$

From Figures 5 and 6 we note that:

- in the first quadrant, the lower bound presents, in both cases, good quality, contrary to the upper bound that seems to be better with the increase of $\delta$;

- in the second quadrant, we observe a clear increase in the quality with the increase of $\delta$, especially in what concerns the upper bound; 
- in the third and fourth quadrants, the quality of the lower bound increases with $\delta$, but it does not happen for the upper bound.

It will be interesting to analyze if the quality of the bounds is related with $\delta$ or, alternatively, with other parameters associated to the $\delta$ order stationarity, as $S_{\delta}$, for example, which is equal, in this study, to 0.529 , when $\delta=\frac{1}{2}$, and 0.6 , when $\delta=2$.

We point out that, by enlarging the plotted region, the bounds seem to be still valid, as happened in the study of the marginal distribution.

The bounds obtained for $F_{\left(X_{1}, X_{2}\right)}\left(x_{1}, x_{2}\right)$ reveal a very good quality, the upper bound in the third and fourth quadrants needing eventually additional attention.

As a final note, we observe that to obtain the empirical distribution function for the vector $\left(X_{1}, X_{2}\right)$ in Figure 6 (here $n=2$ ) it took us $62.5 \%$ more time when compared with the time needed to obtain the same function for the variable $X_{1}$ drawn in the bottom right graphic of Figure 1 (here $n=1$ ). In both graphics we have the same $\delta$-TGARCH $(1,1)$ model and the same number of trajectories of the process simulated. On the contrary, the computation times related to the calculation of the bounds are not significantly different which may be seen as an advantage of the bounding of the distribution of the process when compared with its nonparametric estimation.

\section{Application to Control Charts}

In many situations we are interested in detecting if an observed process $Y$ diverts from a supposed target process $X$. Control charts are used for exactly that purpose. This type of control scheme provides control limits that signal an out-of-control situation if surpassed by the observed process.

In control charts for time series, in particular for $\delta$-TGARCH processes, two main problems arise in contrast with the classical context as we don't have independent observations nor the normality of the observed characteristic. Moreover, the marginal law of the underlying process is unknown ant its non-parametric estimation becomes mandatory. In order to overcome these issues, Severin and Schmid (1999) proposed control charts for standard GARCH processes, namely modified Shewhart control charts with symmetric limits to detect changes in the mean of the process. 
When designing this kind of control charts that allow for asymmetric limits, two distribution functions take on an added importance.

First, the marginal distribution of the target process is used to set the control limits so that, when the process is in in-control, it is equally likely that an observed value falls below the lower control limit $(L C L)$ or above the upper control limit $(U C L)$. More specifically, we assume a Type-I risk $\alpha$ such that $P\left(X_{t}<L C L\right)=$ $P\left(X_{t}>U C L\right)=\alpha / 2$. To evaluate that probability we believe that the bound $F_{Z}\left(x\left[E\left(\sigma_{t}^{\delta}\right)\right]^{-\frac{1}{\delta}}\right)$ can be very relevant due to the exceptional quality present in both symmetric and asymmetric cases.

Second, the distribution function of $\left(X_{1}^{+}, X_{1}^{-}, \ldots, X_{n}^{+}, X_{n}^{-}\right)$is used to evaluate the average run length $(A R L)$, which is the average number of instants that must go by before an out-of-control situation is signaled. Usually a control chart is set so that it has a specific in-control $A R L$ (i.e., the $A R L$ when the observed process is in-control). For example, Severin and Schmid (1999) suggest that, in financial applications using daily stock market values, the in-control $A R L$ should be 20,40 or 60 , because these values correspond to 1,2 or 3 months of stock market activity. The $A R L$ is also used to evaluate the performance control charts, as it is important to have schemes with a high in-control $A R L$ but with a low out-of-control $A R L$. To calculate the $A R L$, the probability of no alarm until time $n$ is needed, which is, in the in-control state,

$$
P\left(-a \leq X_{t} \leq b, t=1, \ldots, n\right)=F_{\left(X_{1}^{+}, X_{1}^{-}, \ldots, X_{n}^{+}, X_{n}^{-}\right)}(b, a, \ldots, b, a)
$$

where $a=-L C L>0$ and $b=U C L>0$. This equality can be extended to the out-of-control state if a relation is established between the observed and the target processes, like a change point model. Here the bounds of the distribution function of $\left(X_{1}^{+}, X_{1}^{-}, \ldots, X_{n}^{+}, X_{n}^{-}\right)$can be used to evaluate the $A R L$, as shown in Gonçalves, Leite and Mendes-Lopes (2013b).

\section{Conclusion}

In this paper we estimate the probability distribution of a power TGARCH process, $X=\left(X_{t}, t \in \mathbb{Z}\right)$, by establishing bounds for their finite dimensional laws.

These bounds for the distribution function are expressed in terms of the distribution function of its generating process and of the parameters of the model. For 
$n \geq 2$, they are established by means of a preliminary bound for the $2 n$-dimensional vector $\left(X_{1}^{+}, X_{1}^{-}, \ldots, X_{n}^{+}, X_{n}^{-}\right)$.

The overall good quality of these theoretical bounds is illustrated by a simulation study with $n=1$ and $n=2$.

The examples presented show that the procedure here proposed is an alternative to the classical estimation of the finite dimensional laws of a process by the empirical distribution functions.

We point out that this probabilistic methodology will be useful, in particular, to evaluate control charts with symmetric or asymmetric bounds for the general class of conditional heteroskedastic processes considered in this study. For some particular models of this wide class and in the context of symmetrical control charts we have shown in Gonçalves, Leite and Mendes-Lopes (2013b) the interest and quality of this methodology.

\section{Acknowledgements}

The authors express their gratitude to the anonymous referees for their valuable suggestions and comments that have significantly improved the paper.

This work is supported by the Centro de Matemática da Universidade de Coimbra (funded by the European Regional Development Fund through the program COMPETE and by the Portuguese Government through the FCT - Fundação para a Ciência e Tecnologia under the project PEst-C/MAT/UI0324/2013).

\section{References}

Ding, Z., Granger, C.W.J. and Engle, R.F. (1993) A long memory property of stock market returns and a new model. Journal of Empirical Finance 1, 83-106.

Francq, C., Wintenberger, O. and Zakoïan, J.M. (2013) GARCH models with positivity constraints: Exponential or log GARCH?. Journal of Econometrics $177,34-46$.

Francq, C. and Zakoïan, J.M. (2010) GARCH Models: Structure, Statistical Inference and Financial Applications. Wiley. 
Gonçalves, E., Leite, J. and Mendes-Lopes, N. (2012) On the probabilistic structure of power threshold generalized ARCH stochastic processes. Statistics and Probability Letters 82, 1597-1609.

Gonçalves, E., Leite, J. and Mendes-Lopes, N. (2013a) On the finite dimensional laws of threshold GARCH processes. Recent Developments in Modeling and Applications in Statistics (Vichi, M., Oliveira, P.E., Temido, M.G., Henriques, C., eds.), Selected Papers of the Statistical Societies, Springer, 237-247.

Gonçalves, E., Leite, J. and Mendes-Lopes, N. (2013b) The ARL of modified Shewhart control charts for conditionally heteroskedastic models. Statistical Papers $54,1-19$.

Gonçalves, E. and Mendes-Lopes, N. (2007) On the distribution of generalized threshold ARCH stochastic processes. International Journal of Pure and Applied Mathematics 35, 397-419.

Pan, J., Wang, H. and Tong, H. (2008) Estimation and tests for power-transformed and threshold GARCH models. Journal of Econometrics 142, 352-378.

Pawlak, M. and Schmid, W. (2001) On the distributional properties of GARCH processes. Journal of Time Series Analysis 22, 339-352.

Severin, T. and Schmid, W. (1999) Monitoring changes in GARCH processes. Allgemeines Statististisches Archiv 83, 281-307.

Taylor, S.J. (2007) Asset Price Dynamics, Volatility, and Prediction. Princeton University Press. 